\title{
Design evolution and properties of superconducting parallel-bar rf-dipole deflecting and crabbing cavities
}

\author{
S. U. De Silva* and J.R. Delayen ${ }^{\dagger}$ \\ Center for Accelerator Science, Department of Physics, Old Dominion University, Norfolk, Virginia 23529, USA, \\ and Accelerator Division, Thomas Jefferson National Accelerator Facility, Newport News, Virginia 23606, USA
}

(Received 7 August 2012; published 22 January 2013)

\begin{abstract}
Deflecting/crabbing cavities serve a variety of purposes in different accelerator applications, primarily in separating a single beam into multiple beams and in rotating bunches for head-on collisions at the interaction point in particle colliders. Deflecting/crabbing cavities are also used for transverse and longitudinal emittance exchange in beams, x-ray pulse compression, and for beam diagnostics. Compact superconducting deflecting/crabbing cavities are under development due to strict dimensional constraints and requirements for higher field gradients with low surface losses. The TEM-like superconducting parallel-bar cavity supports low operating frequencies, thus making the design favorable for many of the deflecting/crabbing cavity applications. The design of the parallel-bar cavity based on cylindrical straight loading elements and rectangular outer conductors has evolved and been adapted to improve the design properties by modifying the design geometry. The improved design with trapezoidalshaped loading elements and cylindrical outer conductor has attractive properties such as low and wellbalanced peak surface fields and high transverse shunt impedance. Additionally, the wide separation of modes in the higher-order mode spectrum and the absence of lower-order mode are advantageous in high current applications. The evolution of the parallel-bar geometry into an rf-dipole geometry is presented with a detailed analysis of the properties for each design.
\end{abstract}

DOI: 10.1103/PhysRevSTAB.16.012004

PACS numbers: 29.27.Ac, 41.85.Ar, 41.85.Ct

\section{INTRODUCTION}

The early application of deflecting/crabbing cavities was in rf deflecting systems designed to separate high energy particle beams [1]. A deflecting cavity system separates a single beam into multiple beams by providing a transverse momentum at the center of each bunch, displacing the bunch off axis at an angle. The corresponding rf phase at which the transverse force is applied is determined by the number of separated beams. The first experimental attempt in designing deflecting cavities was the $2.856 \mathrm{GHz}$ rectangular deflecting cavity that successfully deflected a $150 \mathrm{MeV}$ electron beam at the Mark III linear electron accelerator at Stanford University in 1960 [1]. Following that success, in the early 1960's concurrent work was pursued at CERN [2], SLAC [3], and BNL [4], leading to more advanced designs such as the multicell $\mathrm{TM}_{11}$-type disk loaded waveguide structure [5].

The first superconducting rf deflecting structure was designed at KfK Karlsruhe in collaboration with CERN [6]. The 104-cell standing wave rf particle separator cavity installed at CERN in 1977 was capable of delivering a

\footnotetext{
*pdesilva@odu.edu; sdesilva@jlab.org
}

†jdelayen@odu.edu; delayen@jlab.org

Published by the American Physical Society under the terms of the Creative Commons Attribution 3.0 License. Further distribution of this work must maintain attribution to the author(s) and the published article's title, journal citation, and DOI. deflection in the vertical plane. The deflecting cavity is currently at IHEP. Since then several superconducting and room-temperature deflecting structures have been designed and operated $[7,8]$.

The number of particles colliding at the interaction point is one of the factors contributing to luminosity in a particle collider, where well-overlapped bunches enable the collision of the maximum number of particles in each bunch. The crab crossing concept proposed by Palmer [9] suggests using a crabbing cavity system to increase the luminosity in linacs, and also in collider rings [10]; a crabbing system would allow head-on collision of bunches at the interaction point. The transverse momentum imparted in the opposite direction to the head and tail of each bunch rotates them allowing the overlapped collision. The crabbing cavities are placed on the beam line at zero crossing and operate with a $\pm 90^{\circ}$ synchronous rf phase. A set of crabbing cavities are used after the interaction point to compensate the oscillation which otherwise may lead to beam instabilities. The first crabbing cavity system was developed and installed in 2007 at KEK [11] for the KEKB electronpositron collider. The crabbing cavity operating in $\mathrm{TM}_{110}$-like mode at $509 \mathrm{MHz}$ was the only crabbing cavity system that has been in operation in a particle collider. The possible luminosity upgrade of the LHC [12] has lately drawn attention to the design of compact crabbing cavity systems operating at low frequencies. Some of those designs are the SLAC half-wave spoke resonator crabbing cavity [13], the parallel-bar cavity [14], the Lancaster 
University-Cockroft Institute 4-rod cavity [15], the KEK Kota Cavity [16], and the BNL quarter wave cavity [17].

\section{TYPES OF DEFLECTING/CRABBING CAVITY STRUCTURES}

According to the Panofsky-Wenzel theorem [18,19], under the assumption that the particle traverses the cavity in a straight line at constant velocity, the transverse momentum imparted on the particle is related to the transverse gradient of the longitudinal voltage gain. It can be generated by a TM-type deflecting/crabbing cavity or by a cavity operating in TEM-type mode. Cavity designs with pure TE-type operating mode do not generate a transverse momentum as the net effect from the electric field is canceled by the magnetic field component when integrated along the beam line.

The transverse momentum imparted on the particles in $\mathrm{TM}$ deflecting/crabbing cavities operating in a pure $\mathrm{TM}_{110}$ mode is due to the interaction with the transverse magnetic field as shown in Fig. 1. The cylindrical cross section is usually altered into a squashed geometry in order to remove the degeneracy of the two polarizations of the fundamental deflecting mode. The presence of the beam pipes introduces a transverse electric field that gives an additive effect to the net deflection. The contribution from the transverse electric field to the transverse voltage is small compared to that of the transverse magnetic field at small beam aperture radii. However, at large beam aperture the contribution from the transverse electric field can become comparable to that from the transverse magnetic field.

Most of the deflecting/crabbing cavities designed to date are TM-type cavities which are favorable for higher frequency applications since, for lower frequencies, the designs are fairly large in shape. A number of TM-type deflecting/crabbing cavity designs have been designed, fabricated, and tested to date [20-23], of which the KEK crabbing cavity [11] was the only design that was operational and has been discontinued.

In TEM-type structures, the deflection is produced by the interaction with both electric and magnetic fields. The

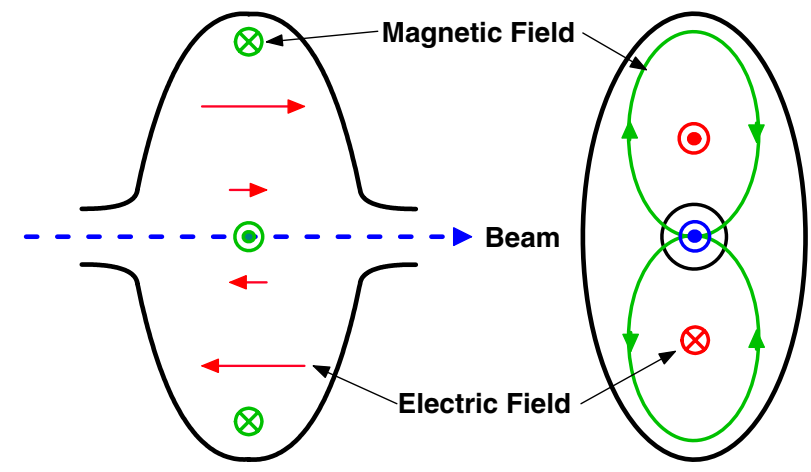

FIG. 1. Field diagram of the deflecting mode in a TM-type design.

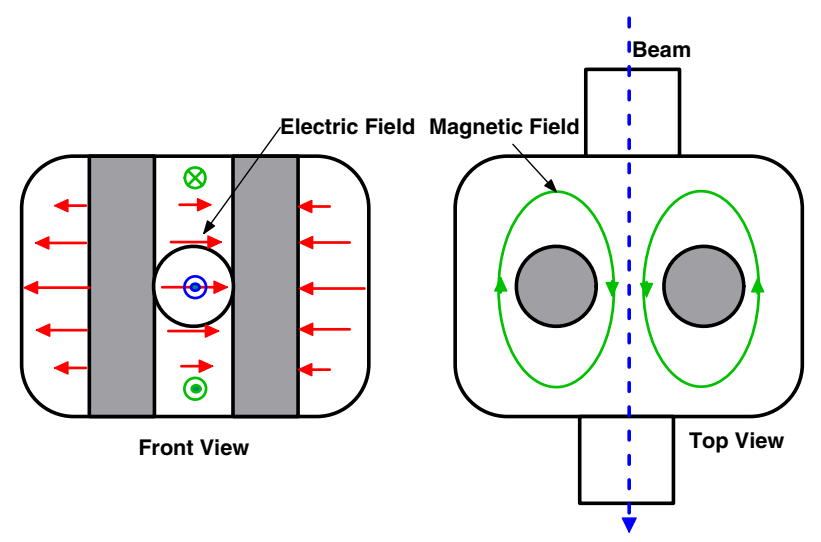

FIG. 2. Field diagram of the deflecting mode in a TEM-type parallel-bar design.

first TEM-type structure is the normal conducting 4-rod $\mathrm{rf}$ separator at CEBAF in Jefferson Lab [7,24], which is used to separate the $6 \mathrm{GeV}$ electron beam for simultaneous delivery to the three experimental halls. The superconducting parallel-bar design [14] is one of the novel compact TEM-type deflecting/crabbing cavities that is currently being considered for a number of applications.

The TEM-type parallel-bar deflecting/crabbing cavity in its simplest form consists of two $\lambda / 2$ TEM parallel lines connecting the top and bottom plates [14]. The number of fundamental modes is determined by the number of TEM parallel lines in the cavity, thus the TEM-type parallel-bar cavity has two fundamental degenerate modes. In the 0-mode the parallel lines oscillate in phase, canceling the transverse field between the lines. In the other degenerate mode (the $\pi$ mode) in which the parallel lines oscillate out of phase, the transverse electric field between the lines, produces the deflection as shown in Fig. 2. The magnetic field is zero on the midplane along the beam line and maximum at the top and bottom planes.

\section{PARAMETERS OF DESIGN OPTIMIZATION}

The transverse momentum $\left(\vec{p}_{t}\right)$ experienced by a particle passing along the beam axis, shown in Eq. (1), is a direct result of the interaction between the particle and the transverse electric and magnetic field components present in the cavity:

$$
\vec{p}_{t}=\int_{-\infty}^{\infty} \vec{F}_{t} \mathrm{~d} t=\frac{q}{v} \int_{-\infty}^{+\infty}\left[\vec{E}_{t}+\left(\vec{v} \times \vec{B}_{t}\right)\right] \mathrm{d} z,
$$

where $\vec{F}_{t}$ is the transverse Lorentz force, $q$ is the charge of the particle, $\vec{v}$ is the velocity of the particle, and $\vec{E}_{t}$ and $\vec{B}_{t}$ are the corresponding transverse electric and magnetic field components.

The Panofsky-Wenzel theorem $[18,19]$ relates the transverse momentum acquired by the particle to the gradient of the longitudinal electric field along the beam axis under the 
assumption that the particle travels at a constant velocity on a straight line:

$$
\begin{aligned}
\vec{p}_{t} & =-i \frac{q}{\omega} \int_{-\infty}^{+\infty} \vec{\nabla}_{t} E_{z} \mathrm{~d} z \\
& =-i \frac{q}{\omega} \lim _{r_{0} \rightarrow 0} \frac{1}{r_{0}} \int_{-\infty}^{+\infty}\left[E_{z}\left(r_{0}, z\right)-E_{z}(0, z)\right] \mathrm{d} z,
\end{aligned}
$$

where $\omega$ is the frequency of the deflecting mode, $r_{0}$ is the transverse offset in the direction of the deflection from the beam axis, and $E_{z}$ is the longitudinal electric field component at the offset $r_{0}$. This theorem is valid for any type of deflecting mode of any geometry.

The entire length along the beam line is considered in determining the transverse voltage as a small amount of the transverse field extends into the beam apertures at the ends of the cavity.

For a pure TEM-type parallel-bar deflecting/crabbing cavity, the transverse field components on axis that contribute to the net deflection are of the form [14]

$$
\begin{aligned}
& \vec{E}_{t}=\vec{E}_{x}(z) \cos (\omega t), \\
& \vec{H}_{t}=\vec{H}_{y}(z) \sin (\omega t) .
\end{aligned}
$$

Hence, for a particle with velocity $\beta=1$, the transverse voltage is given by

$$
V_{t}=\int_{-\infty}^{+\infty}\left[E_{x}(z) \cos \left(\frac{\omega z}{c}\right)+c B_{y} \sin \left(\frac{\omega z}{c}\right)\right] \mathrm{d} z .
$$

The optimal effective length of the structure along the beam line for a particle traveling at the velocity of light will be of the order of $n \lambda / 2$, where $\lambda$ is the free-space wavelength of the deflecting mode and $n$ is the number of pairs of bars, and we are defining the transverse deflecting field by

$$
E_{t}=\frac{V_{t}}{n \lambda / 2}
$$

where $V_{t}$ is the transverse voltage. In all the geometries presented in this paper $n=1$, and therefore

$$
E_{t}=\frac{V_{t}}{\lambda / 2}
$$

The half wavelength was chosen as the reference length in the definition of the transverse deflecting field instead of the cavity length since $\lambda / 2$ is constant whereas the cavity length is a free optimization parameter that may be different between various designs.

In deflecting/crabbing structures, the transverse momentum acquired by a particle is proportional to the transverse voltage, and therefore to the peak surface fields. However, in superconducting structures, the peak surface electric and magnetic fields cannot exceed certain values. The peak surface electric field $\left(E_{p}\right)$ is limited by the field emission $[25,26]$ at high field gradients. The contaminant particles or any protrusions present on the surface give rise to field enhancement at localized high electric field regions, emitting electrons from the surface. The emitting electrons traveling with the $\mathrm{rf}$ field may collide with the cavity surface, depositing heat on the cavity surface. The extreme electron currents may lead to a thermal breakdown due to excess heating also reducing the quality factor $\left(Q_{0}\right)$ exponentially, hence limiting the achievable peak surface field. Because of the advanced cleaning and chemical processing techniques in treating the cavity surfaces, at present the superconducting cavities can reach maximum tolerable peak electric field levels in excess of $50 \mathrm{MV} / \mathrm{m}$.

The peak surface magnetic field $\left(B_{p}\right)$ for type II superconductors is confined by the theoretical limit of lower critical magnetic field $\left(H_{c 1}\right)$, which for $\mathrm{Nb}$ is $170 \mathrm{mT}$. However, the achievable peak surface magnetic field in superconducting cavities is often lower than the theoretical one, reducing the reliable field level to be $\sim 100 \mathrm{mT}$. Any defects present on the cavity surface in high magnetic field regions can lead to a local increase of the temperature resulting in excess power dissipation and eventually thermal breakdown $[25,26]$.

Because of the limitations in the peak surface fields, the TEM-type superconducting deflecting/crabbing cavity design presented here was optimized to minimize the corresponding ratios of $E_{p} / E_{t}$ and $B_{p} / E_{t}$ in order to maximize the net deflection while keeping the surface fields at a minimum. The peak surface field ratio given by $B_{p} / E_{p}$ is equally important in designing superconducting cavities with well-balanced peak surface fields. A higher peak field ratio could result a high peak surface magnetic field or a very low peak surface electric field and vice versa for a low peak field ratio. In either case the operating voltage of the cavity may be limited by just one of the peak surface field limits mentioned above. Therefore it is desirable to balance



FIG. 3. Parallel-bar geometry with a rectangular-shaped outer conductor and cylindrical loading elements. 


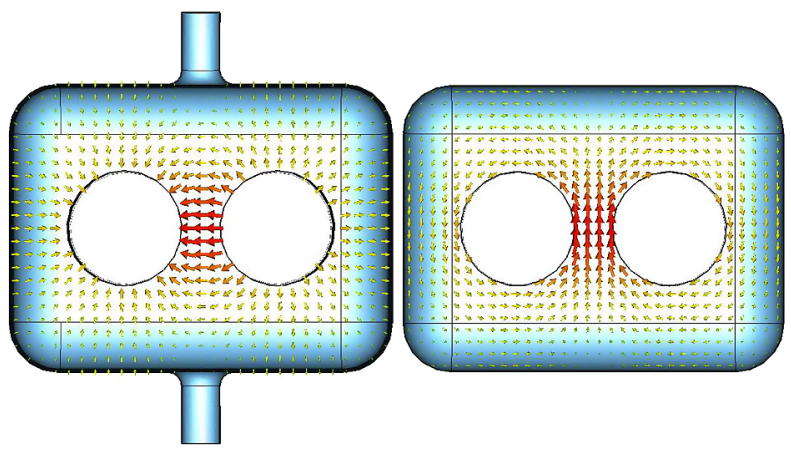

FIG. 4. Electric field (left) on the midplane and magnetic field (right) on the top plane for the fundamental deflecting mode.

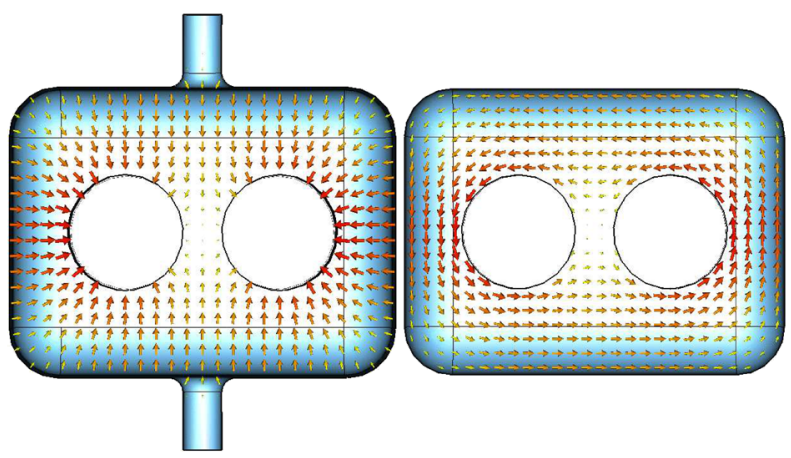

FIG. 5. Electric field (left) on the midplane and magnetic field (right) on the top plane for the fundamental accelerating mode.

the peak field ratio such that the operating limits are met simultaneously. Furthermore, whether the cavities will be used in deflecting or crabbing applications, they will necessarily be in small number. On the other hand, they must achieve their design performance in order to be operational. Thus, the peak surface electric and magnetic field must be limited to values that can be reached with a high probability, sufficiently below the highest that have been

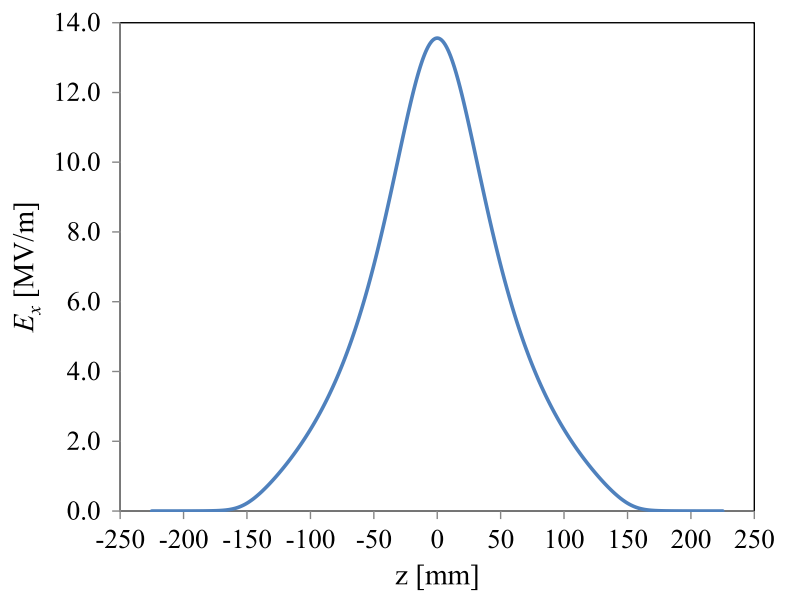

demonstrated at low frequency [27]. Given the state of the art we have chosen $E_{p} \simeq 35 \mathrm{MV} / \mathrm{m}$ and $B_{p} \simeq 70 \mathrm{mT}$ [and therefore $\left.B_{p} / E_{p} \simeq 2 \mathrm{mT} /(\mathrm{MV} / \mathrm{m})\right]$ as design values. This ratio would be slightly less than $2 \mathrm{mT} /(\mathrm{MV} / \mathrm{m})$ for cavities operating at $4.2 \mathrm{~K}$ and slightly more for cavities operating at $\sim 2 \mathrm{~K}$.

The other parameter of interest is the product of transverse shunt impedance $\left(R_{t}\right)$ and surface resistance $\left(R_{s}\right)$ that relates to the power dissipation on the cavity surface $\left(P_{\text {diss }}\right)$ as shown in

$$
R_{t}=\frac{V_{t}^{2}}{P_{\text {diss }}}
$$

with the geometrical factor $(G)$ for any cavity geometry given by

$$
G=Q_{0} R_{S}
$$

where $Q_{0}$ is the intrinsic quality factor defined as

$$
Q_{0}=\frac{\omega U}{P_{\mathrm{diss}}},
$$

and $U$ is the stored energy content in the cavity.

Furthermore, the transverse $[R / Q]_{t}$ is determined as

$$
\left[\frac{R}{Q}\right]_{t}=\frac{V_{t}^{2}}{\omega U}
$$

The $R_{t} R_{s}$ for a given deflecting voltage $\left(V_{t}\right)$ needs to be maximized in order to minimize the power dissipation and is given by

$$
R_{t} R_{s}=\frac{V_{t}^{2}}{P_{\mathrm{diss}}} \frac{G}{Q_{0}}=\frac{V_{t}^{2}}{\omega U} \frac{\omega U}{P_{\mathrm{diss}}} \frac{G}{Q_{0}}=\left[\frac{R}{Q}\right]_{t} G
$$

\section{DESIGN OF PARALLEL-BAR CAVITY}

The TEM-type parallel-bar structure shown in Fig. 3 consists of two cylindrical parallel bars of length $\lambda / 2$,

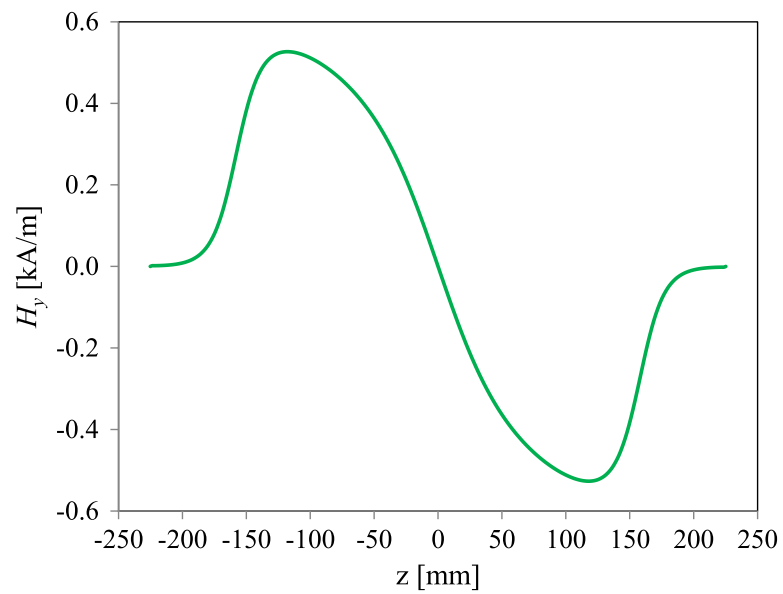

FIG. 6. Transverse electric and magnetic field components along the beam axis, at an energy content of $1 \mathrm{~J}$ in a $499 \mathrm{MHz}$ parallel-bar cavity shown in Fig. 3. 
perpendicular to the beam line passing between the bars. The cavity shape with the rectangular-shaped outer conductor has a length of $\lambda / 2$ and the width is independent of the wave length. As mentioned earlier the design has two fundamental degenerate modes.

The electric field distribution of the deflecting mode ( $\pi$-mode) is shown in Fig. 4. The transverse electric field is concentrated between the parallel bars, where the magnetic field is maximum on the top and bottom plane as shown in Fig. 4 and the electric field is maximum on the midplane between the parallel bars.

In the 0-mode the transverse electric field is canceled between the bars (Fig. 5), however there is a longitudinal component remaining near the ends of the cavity so this mode would operate as an accelerating mode. The transverse field is stronger between the bars and the sidewalls of the cavity. Likewise the magnetic field circles enclosing both the parallel bars as shown in Fig. 5 .

In a $499 \mathrm{MHz}$ cavity the degeneracy of the two fundamental modes ( $\pi$-mode and 0 -mode) is slightly removed by $\sim 1 \mathrm{MHz}$ with the inclusion of the beam pipe. The modes are further separated by rounding the edges on the top and the bottom plane. The rounded edges give rise to a magnetic field in the vertical direction between the parallel bars and the front and end plates of the rectangular-shaped cavity. Therefore the parallel-bar deflecting/crabbing cavity has both electric and magnetic field components along the beam axis. The corresponding field components at a normalized stored energy of $1 \mathrm{~J}$ are shown in Fig. 6 . The net deflection seen by a particle with velocity $\beta=v / c=1$ is determined as in Eq. (5). The main contribution to the net deflection is by the horizontal electric field component $\left(E_{x}\right)$ while the vertical magnetic field $\left(H_{y}\right)$ component opposes the net deflection; however the effect from the magnetic field component is comparatively small.

The TEM-type parallel-bar cavity has no on-axis longitudinal electric field component. The longitudinal electric

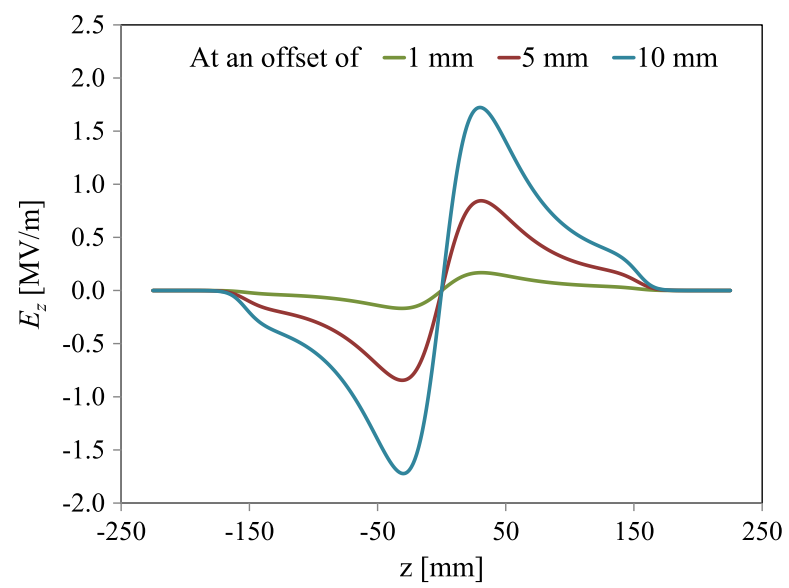

FIG. 7. Longitudinal electric field component at an offset of 1, 5 , and $10 \mathrm{~mm}$ at an energy content of $1 \mathrm{~J}$ in a $499 \mathrm{MHz}$ parallelbar cavity shown in Fig. 3 . field increases transversely in horizontal direction as shown in Fig. 7. The off-axis longitudinal electric field can also be used to determine the net deflection using Eq. (2). The net deflection calculated in the direct integral method and using the Panofsky-Wenzel theorem are in agreement within $0.07 \%$.

\section{A. Evolution of the parallel-bar cavity geometry into the rf-dipole geometry}

A series of parallel-bar cavity geometries with different outer conductors and parallel loading elements (Fig. 8) have been analyzed and compared in identifying a design
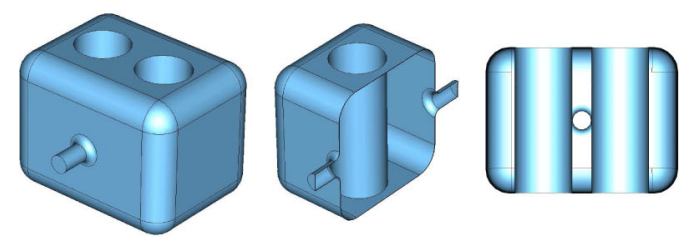

Design (A)
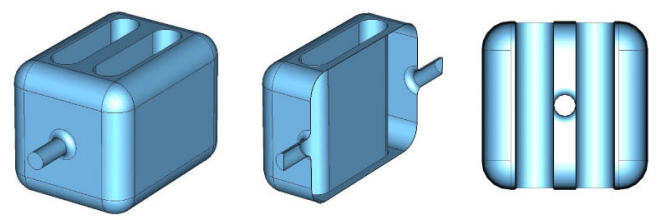

Design (B)
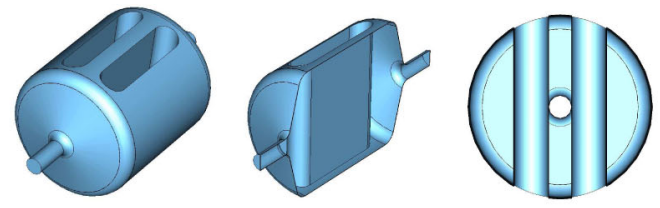

Design (C)
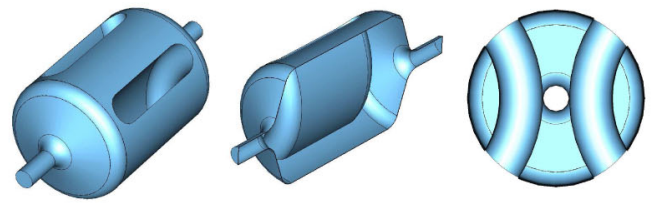

Design (D)
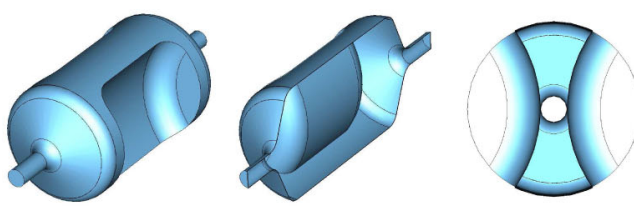

Design (E)
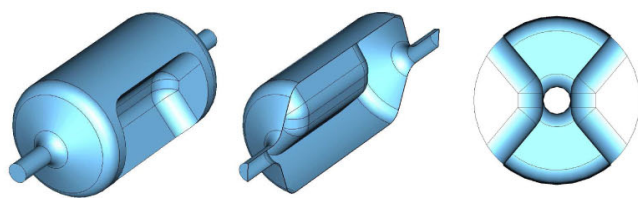

Design (F)

FIG. 8. Evolution of the parallel-bar cavity geometry into the rf-dipole geometry and cross sections of each design. 



FIG. 9. Ratios of peak surface electric field $\left(E_{p}\right)$ and magnetic field $\left(B_{p}\right)$ to the transverse electric field $\left(E_{t}\right)$ with varying radius of cylindrical-shaped parallel bars [Design (A)].

with optimal properties [28,29]. The design properties for each design are analyzed using the 3D eigenmode simulator CST MICROWAVE STUDIO [30]. The operational frequency for each design is $499 \mathrm{MHz}$ with a fixed beam aperture diameter of $40 \mathrm{~mm}$. The parallel-bar geometry is adapted by changing the rectangular-shaped outer conductor into a cylindrical-shaped design, specifically to increase the mode separation in the higher-order mode (HOM) spectrum [31,32]. Furthermore, the parallel loading elements are adapted in such a way to maximize the net deflection with low and well-balanced peak surface fields.

The parallel-bar cavity design proposed by the analytical model [14] shown by Design (A) in Fig. 8 has cylindrical loading elements in a rectangular-shaped outer conductor. The length of the cavity is $\lambda / 2$, however the cavity height is slightly greater than $\lambda / 2$ with the adjustments made to achieve the design frequency of $499 \mathrm{MHz}$, due to the rounded edges of the cavity. In this design the radius of the cylindrical loading elements is optimized with a fixed outer conductor in order to maximize the net deflection and minimize peak surface fields, by minimizing the ratios of $E_{p} / E_{t}$ and $B_{p} / E_{t}$ as shown in Fig. 9.

The peak surface fields for this design are shown in Fig. 10, where peak electric field is concentrated in the middle of the bars and the peak magnetic field is highest on the top and bottom surfaces of the bars and cavity. At a smaller radius both electric and magnetic peak fields between the parallel bars are high, resulting in higher ratios of $E_{p} / E_{t}$ and $B_{p} / E_{t}$. As the radius is increased the design properties improve due to the increase in the transverse electric field component along the beam line and the decreasing surface fields.

The design properties of the parallel-bar deflecting cavity are shown in Table I. The frequency separation between the two fundamental modes of $11 \mathrm{MHz}$ is achieved by rounding the cavity edges as the beam line ports contribute little to the frequency separation. The low mode separation could make the damping of the higher-order modes more challenging. It should be noted however that the deflecting mode is always the lowest frequency mode. The resultant peak surface field ratios to the transverse electric field are $E_{p} / E_{t}=3.45$ and $B_{p} / E_{t}=11.47 \mathrm{mT} /(\mathrm{MV} / \mathrm{m})$. Therefore a cavity required to deliver a transverse voltage of $3 \mathrm{MV}$ needs to operate at a peak surface electric field of $E_{p}=34.5 \mathrm{MV} / \mathrm{m}$ and at a peak surface magnetic field of $B_{p}=114.7 \mathrm{mT}$, which is somewhat higher than the achievable practical limit. In pure TEM modes $E_{p} / H_{p}=$ $Z_{0} \simeq 377 \Omega$, the impedance of vacuum (irrespective of geometry) or $B_{p} / E_{p}=3.33 \mathrm{mT} /(\mathrm{MV} / \mathrm{m})$. In order to achieve a better balanced peak surface field ratio of 2.0 the peak surface magnetic field would then need to be reduced at the expense of an increased peak surface electric field.

The $R_{t} R_{s}$ is determined as given in Eq. (12) and has quadratic dependence with the radius of the cylindrical parallel bars as shown in Fig. 11. It can be noticed that the radius that maximizes the shunt impedance is close to the one that minimizes the peak surface fields.
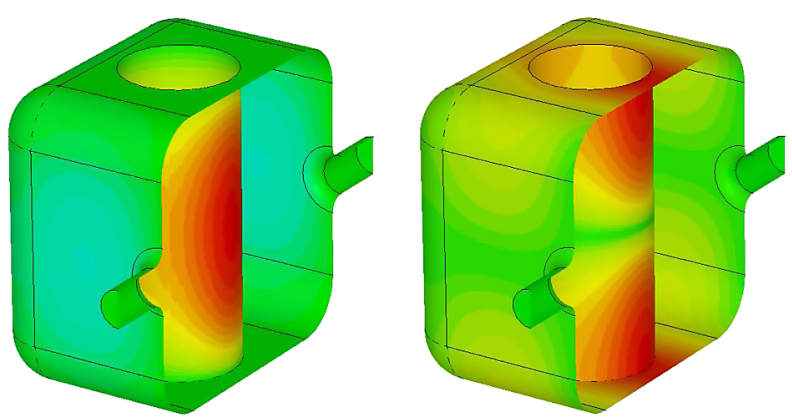

FIG. 10. Surface electric (left) and magnetic (right) fields in the parallel-bar design with cylindrical loading elements [Design (A)]. 
TABLE I. Properties of the geometries shown in Fig. 8.

\begin{tabular}{|c|c|c|c|c|c|c|c|}
\hline Parameter & & & & & & & Units \\
\hline Frequency of $\pi$ mode & 499.0 & 499.0 & 499.0 & 499.0 & 499.0 & 499.0 & $\mathrm{MHz}$ \\
\hline Frequency of 0 mode & 509.7 & 517.4 & 660.4 & 860.4 & 1022.0 & 1036.1 & $\mathrm{MHz}$ \\
\hline Frequency of nearest mode & 509.7 & 517.4 & 626.1 & 763.5 & 754.3 & 777.0 & $\mathrm{MHz}$ \\
\hline$\lambda / 2$ of $\pi$ mode & 300.4 & 300.4 & 300.4 & 300.4 & 300.4 & 300.4 & $\mathrm{~mm}$ \\
\hline Cavity length & 300.4 & 406.5 & 424.0 & 400.0 & 450.0 & 440.0 & $\mathrm{~mm}$ \\
\hline Cavity height & 304.3 & 304.6 & $\cdots$ & $\ldots$ & $\cdots$ & $\cdots$ & $\mathrm{mm}$ \\
\hline Cavity width & 400.0 & 300.0 & $\cdots$ & $\cdots$ & $\cdots$ & $\cdots$ & $\mathrm{mm}$ \\
\hline Cavity diameter & $\cdots$ & $\cdots$ & 318.0 & 272.8 & 250.5 & 241.2 & $\mathrm{~mm}$ \\
\hline Aperture diameter & 40.0 & 40.0 & 40.0 & 40.0 & 40.0 & 40.0 & $\mathrm{~mm}$ \\
\hline Bar length & $\cdots$ & 286.5 & 274.0 & 260.0 & 295.0 & 260.0 & $\mathrm{~mm}$ \\
\hline Bar diameter/width & 120.0 & 70.0 & 60.0 & 65.0 & $\cdots$ & $\cdots$ & $\mathrm{mm}$ \\
\hline Bar height/curved height & 304.3 & 304.6 & 157.7 & 262.4 & 204.0 & $\cdots$ & $\mathrm{mm}$ \\
\hline Bar inner height & $\cdots$ & $\cdots$ & $\cdots$ & $\cdots$ & $\cdots$ & 50.0 & $\mathrm{~mm}$ \\
\hline Angle & $\cdots$ & $\cdots$ & $\cdots$ & $\cdots$ & $\cdots$ & 50.0 & $\operatorname{deg}$ \\
\hline Deflecting voltage $\left(V_{t}^{\mathrm{a}}\right)$ & 0.30 & 0.30 & 0.30 & 0.30 & 0.30 & 0.30 & MV \\
\hline Peak electric field $\left(E_{p}{ }^{\mathrm{a}}\right)$ & 3.45 & 1.83 & 2.08 & 2.55 & 2.85 & 2.86 & $\mathrm{MV} / \mathrm{m}$ \\
\hline Peak magnetic field $\left(B_{p}{ }^{\mathrm{a}}\right)$ & 11.47 & 6.05 & 6.47 & 5.42 & 5.12 & 4.38 & $\mathrm{mT}$ \\
\hline$B_{p} / E_{p}$ & 3.33 & 3.30 & 3.12 & 2.12 & 1.80 & 1.53 & $\mathrm{mT} /(\mathrm{MV} / \mathrm{m})$ \\
\hline Energy content $\left(U^{\mathrm{a}}\right)$ & 0.049 & 0.030 & 0.032 & 0.033 & 0.036 & 0.029 & $\mathrm{~J}$ \\
\hline Geometrical factor $(G)$ & 80.9 & 67.0 & 67.4 & 87.7 & 94.2 & 105.9 & $\Omega$ \\
\hline$[R / Q]_{t}$ & 591.7 & 935.9 & 887.3 & 866.8 & 807.7 & 982.5 & $\Omega$ \\
\hline$R_{t} R_{s}$ & $4.8 \times 10^{4}$ & $6.3 \times 10^{4}$ & $6.0 \times 10^{4}$ & $7.6 \times 10^{4}$ & $7.6 \times 10^{4}$ & $1.0 \times 10^{5}$ & $\Omega^{2}$ \\
\hline
\end{tabular}

${ }^{\mathrm{a}}$ At $E_{t}=1 \mathrm{MV} / \mathrm{m}$.

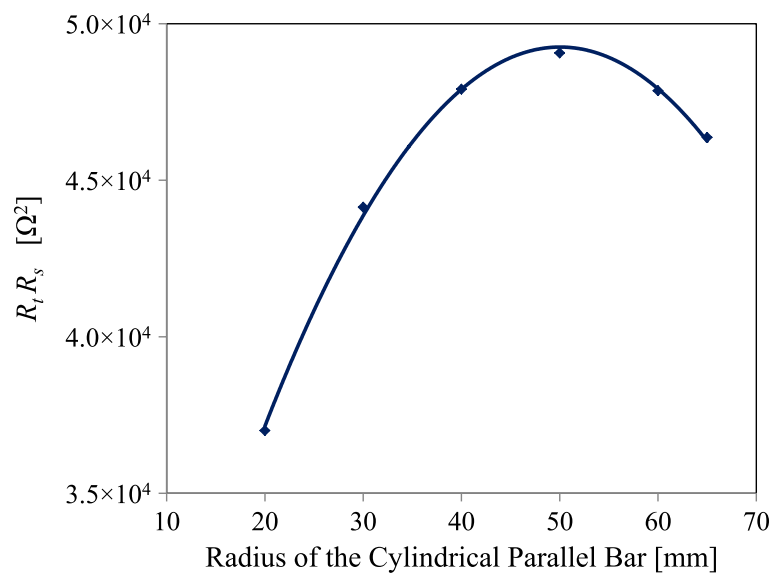

FIG. 11. Product of transverse shunt impedance $\left(R_{t}\right)$ and surface resistance $\left(R_{s}\right)$ with varying radius of cylindrical-shaped parallel bars [Design (A)].
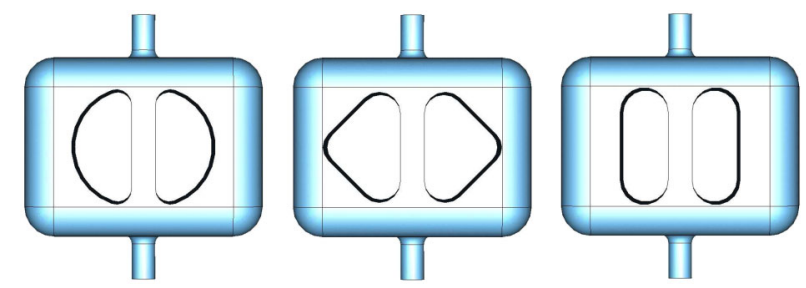

FIG. 12. Parallel-bar cavity designs with different cross sections
It is also clear that the effective deflecting length is equally important in increasing the resultant deflection in the parallel-bar deflecting/crabbing cavity, due to the localized transverse fields between the parallel loading elements. Therefore the peak surface fields for a given deflecting field can be reduced by increasing the effective deflecting length along the beam line. Three design structures shown in Fig. 12 were analyzed in order to optimize the effective length of deflection [33]. The designs have identical rectangular outer conductor as in Design (A), while modifying the parallel-bar orientation to maximize the net deflection. In each design the cross section orientation is maximized to achieve the largest possible effective length, also curving adequately to minimize higher field concentration on edges, enabling more distributed surface

TABLE II. Properties of parallel-bar cavities with different cross sections shown in Fig. 12.

\begin{tabular}{lcccc}
\hline \hline Parameter & 00 & 00 & 00 & \multirow{2}{*}{ Units } \\
\hline$E_{p}{ }^{\mathrm{a}}$ & 2.29 & 2.3 & 2.21 & $\mathrm{MV} / \mathrm{m}$ \\
$B_{p}{ }^{\mathrm{a}}$ & 5.95 & 5.96 & 5.75 & $\mathrm{mT} /(\mathrm{MV} / \mathrm{m})$ \\
{$[R / Q]_{t}$} & 1043.5 & 969.0 & 992.5 & $\Omega$ \\
$G$ & 68.3 & 69.3 & 69.4 & $\Omega$ \\
$R_{t} R_{s}$ & $7.1 \times 10^{4}$ & $6.7 \times 10^{4}$ & $6.9 \times 10^{4}$ & $\Omega^{2}$ \\
\hline \hline
\end{tabular}

${ }^{\mathrm{a}}$ At $E_{t}=1 \mathrm{MV} / \mathrm{m}$. 


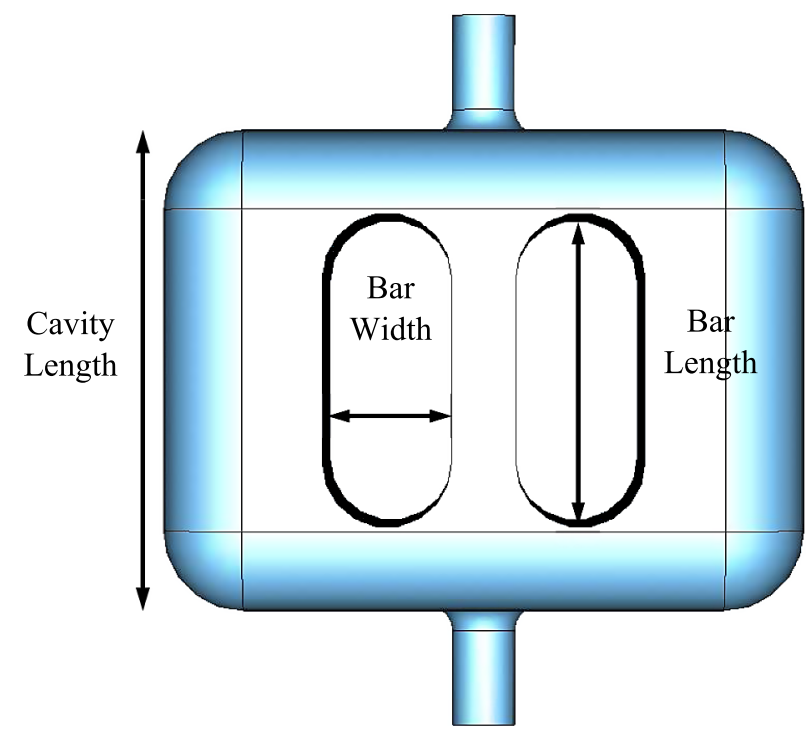

FIG. 13. Design parameters of optimization for the parallel-bar design with rectangular outer conductor and race-track-shaped loading elements [Design (B)].

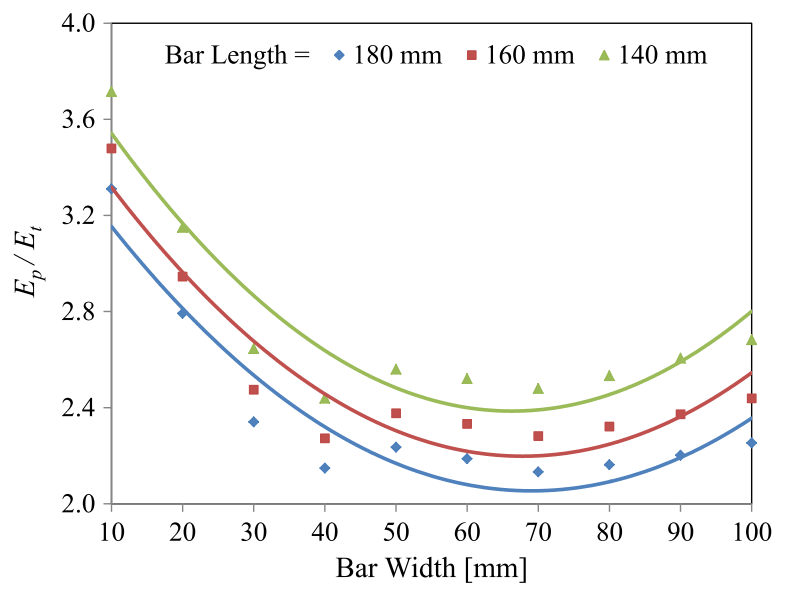

fields. All three designs with increased effective deflecting length have improved properties compared to the parallelbar cavity with cylindrical loading elements and also have more uniform field between the parallel bars along the beam line.

The design properties are comparable as shown in Table II between the designs with half-circular-shaped and triangular-shaped loading elements. However, the design with race-track-shaped loading elements is preferable in reducing peak surface fields with further optimizations described below.

The parallel-bar design with a race-track-shaped loading element in a rectangular-shaped outer conductor [Design (B)] is further improved by varying bar width and the bar length, which are the key optimizing parameters for this design as shown in Fig. 13.

As seen earlier the longer effective deflecting length gives a higher net deflection and the bar width optimizes the spread of the surface electric field on the bar as well as the surface magnetic field on the top and the bottom surfaces in the cavity. The dependence of design properties on the bar width and length of the parallel-bar cavity is
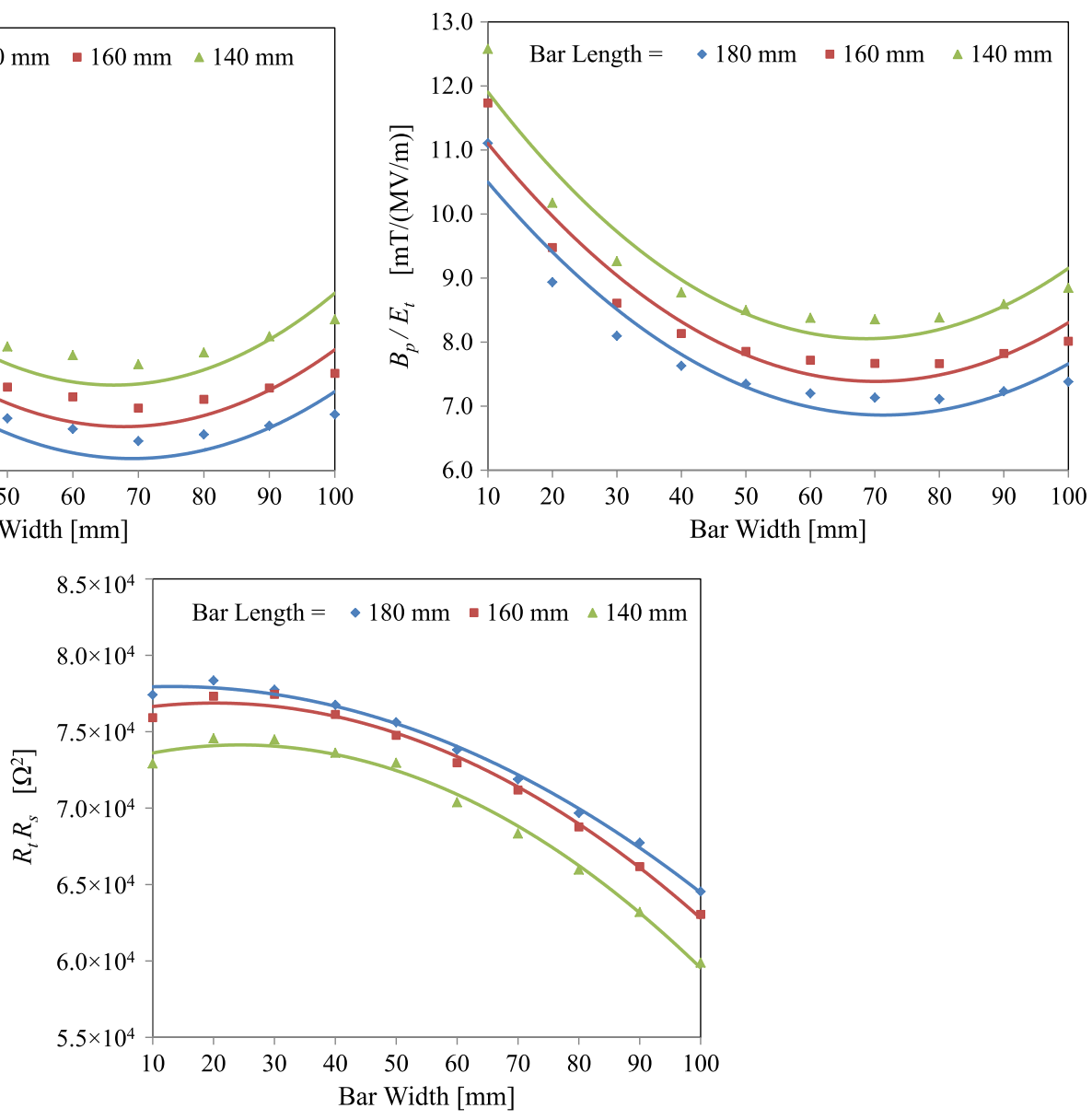

FIG. 14. Ratios of peak surface electric field $\left(E_{p}\right)$ and magnetic field $\left(B_{p}\right)$ to the transverse electric field $\left(E_{t}\right)$ and $R_{t} R_{s}$ with varying bar width and bar length for race-track-shaped parallel bars [Design (B)]. 


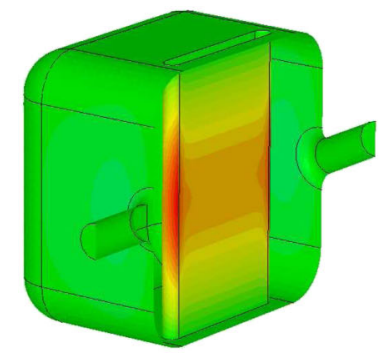

(a)
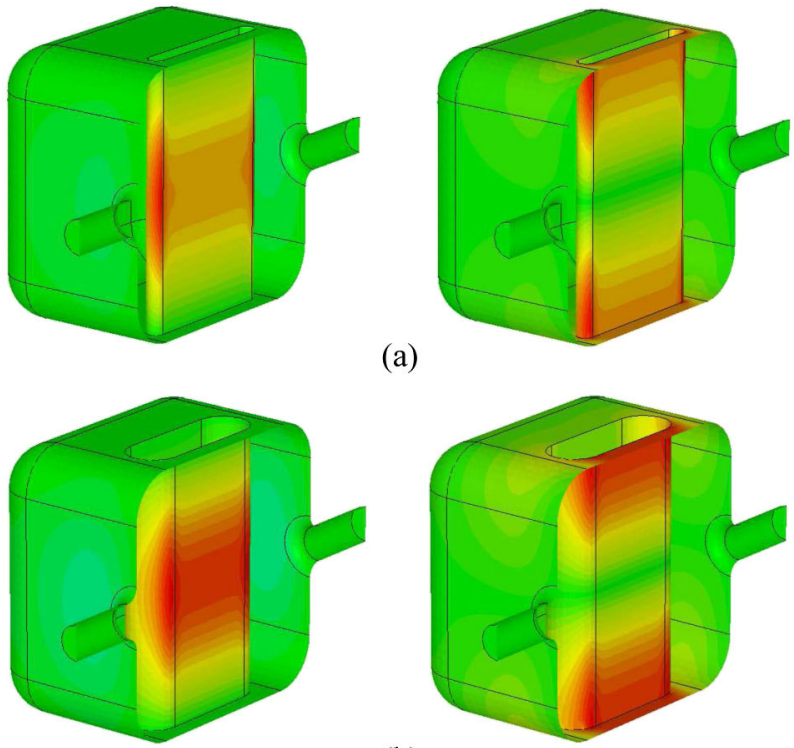

(b)
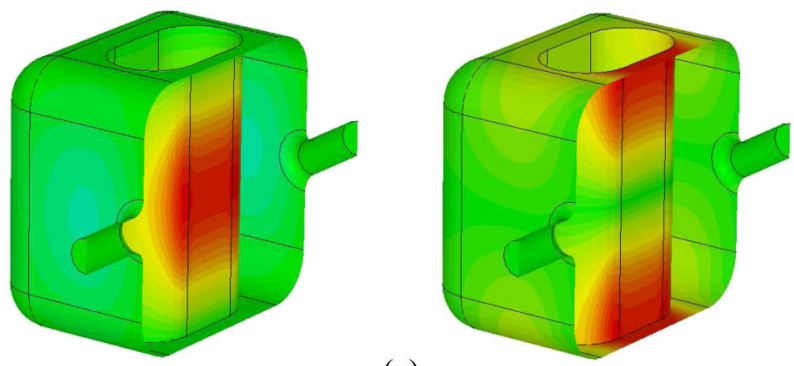

(c)

FIG. 15. Surface electric (left) and magnetic (right) field at bar widths of (a) $20 \mathrm{~mm}$, (b) $60 \mathrm{~mm}$, and (c) $100 \mathrm{~mm}$.

shown in Fig. 14. The ratio $E_{p} / E_{t}$ decreases with the increasing bar length. The width of the race-track-shaped loading elements optimizes the spread of the surface fields as shown in Fig. 15. The strong peak fields at the edges of narrow parallel bars increase both $E_{p} / E_{t}$ and $B_{p} / E_{t}$. In the opposite, wider parallel bars with rounding of the edges reduce the achieved net deflection. The $R_{t} R_{s}$ drops rapidly with the increasing bar width.

In the parallel-bar cavity the transverse electric field is concentrated between the two parallel bars, therefore the bar length can be further optimized with the cavity length, by increasing both bar length and the cavity length. The optimum effective deflecting length is when the bar length is in the order of $\lambda / 2$ as shown in Fig. 16.

The final design properties of the optimized parallel-bar cavity with a rectangular outer conductor and a racetrack-shaped loading elements are given in Table I. The design geometry shows significant improvement on both peak surface electric and magnetic fields of $50 \%$. The peak surface fields are substantially reduced; however, this geometry still has a peak field ratio of $B_{p} / E_{p}=$ $3.3 \mathrm{mT} /(\mathrm{MV} / \mathrm{m})$, higher than desirable. The width is reduced substantially making the design more compact. However, the mode separation between the fundamental modes is still only $\simeq 18 \mathrm{MHz}$. Also the large flat surfaces make the design prone to deformations due to radiation pressure and sensitivity to liquid helium pressure fluctuations. This can lead to a mixing of modes with a longitudinal electric field present in the fundamental deflecting mode [34].

The parallel-bar cavity design is further modified as in Design (C) with a cylindrical outer conductor, keeping the race-track-shaped loading elements, in order to increase the mode separation. The design also incorporates sloped end plates for efficient chemical processing of the cavity inner surface and increased stiffness. The bar width, bar length, and cavity length are optimized in a similar manner as in Design (B). The dependence of the peak field ratios of $E_{p} / E_{t}, B_{p} / E_{t}$, and $R_{t} R_{s}$ is shown in Fig. 17.

The properties of this design are shown in Table I. The frequency is determined mostly by the cavity radius and the transverse dimensions are somewhat larger compared to the respective design with rectangular outer conductor. The design with the cylindrical outer conductor
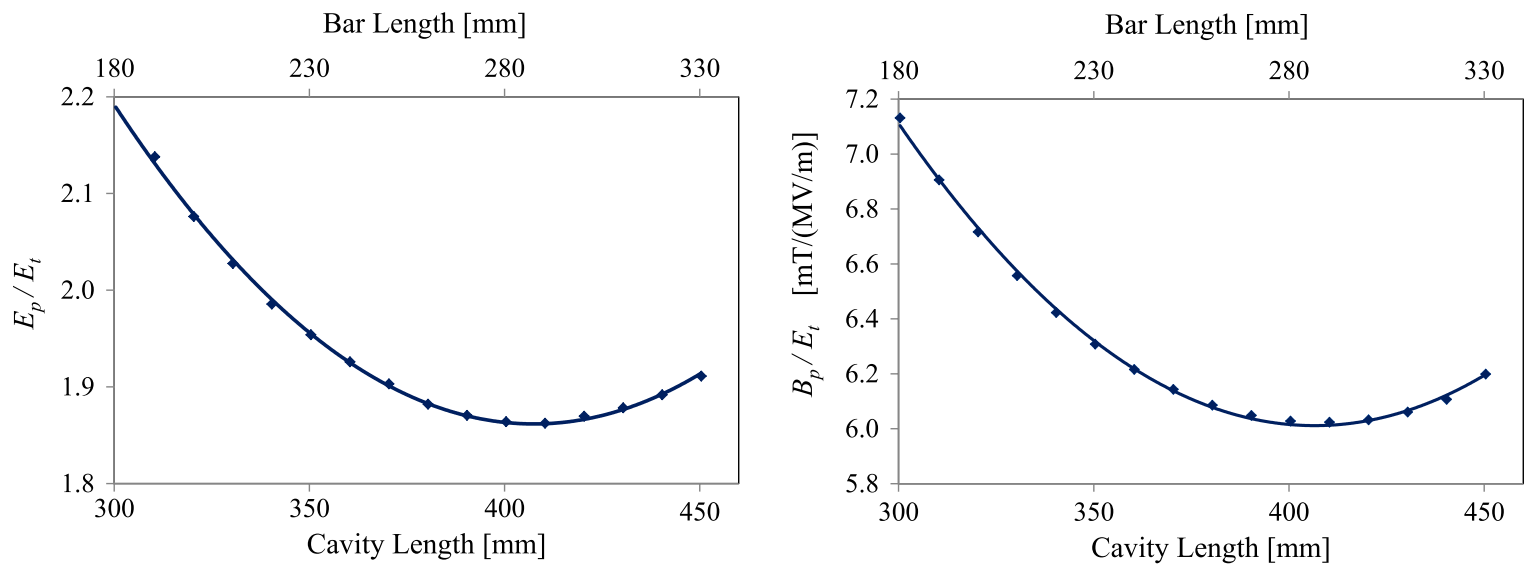

FIG. 16. Ratios of peak surface electric field $\left(E_{p}\right)$ and magnetic field $\left(B_{p}\right)$ to the transverse electric field $\left(E_{t}\right)$ with varying cavity length for race-track-shaped parallel bars [Design (B)]. 

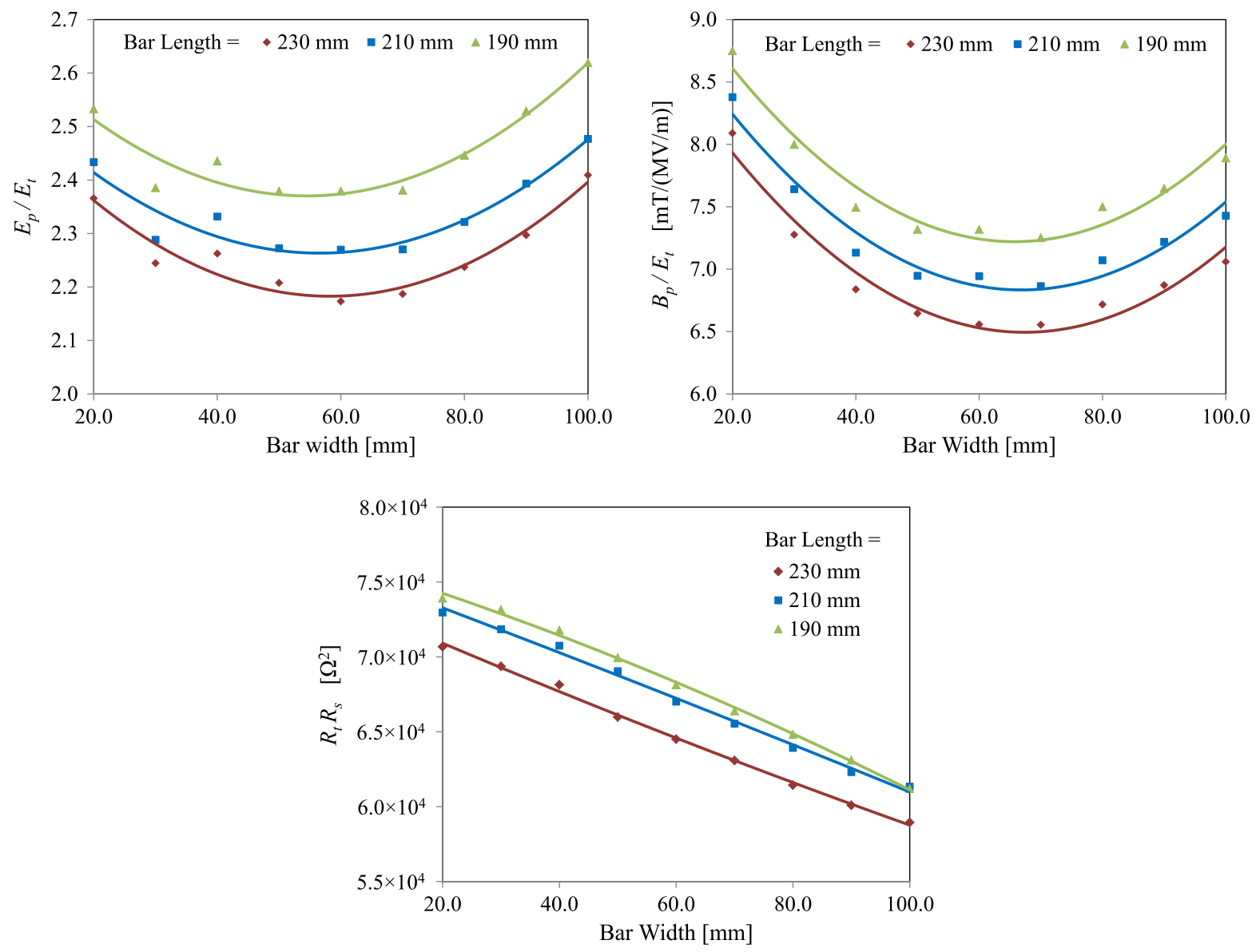

FIG. 17. Ratios of peak surface electric field $\left(E_{p}\right)$ and magnetic field $\left(B_{p}\right)$ to the transverse electric field $\left(E_{t}\right)$ and $R_{t} R_{s}$ with varying bar width and bar length for race-track-shaped parallel bars with cylindrical outer conductor [Design (C)].

has a significantly improved mode separation between the two fundamental modes of $127 \mathrm{MHz}$ compared to the $\simeq$ $18 \mathrm{MHz}$ in the previous design. This geometry also has an increasing mode separation in the higher-order modes spectrum. One limitation in this design is that it still has a high peak surface magnetic field and a far-from-optimal $B_{p} / E_{p}$. Since the parallel bars are constraining the volume for the magnetic field, it is desirable to curve the parallel loading elements to reduce the peak surface magnetic field by increasing the magnetic field volume. The $R_{t} R_{s}$ in Design (C) decreases as the bar width increases, but with a smaller drop compared to Design (B) due to the difference in the field content.

This design is further optimized with curved loading elements as shown in Fig. 8: Design (D). The main optimizing parameters are the bar width and the curvature of the parallel bars, as shown in Fig. 18. The effect of the curving radius and the width of the parallel bars on $E_{p} / E_{t}$ and $B_{p} / E_{t}$ is shown in Fig. 19. As the curvature radius is reduced the peak surface magnetic field decreases and the surface electric field increases. At very high curvatures (i.e. $>400 \mathrm{~mm}$ ) the design properties are similar to those of the straight parallel bars. Also $E_{p} / E_{t}$ and $B_{p} / E_{t}$ are independent of the optimum bar width for varying curvature radius. This clearly shows that the curvature can be used to balance the peak surface electric and magnetic fields. The $R_{t} R_{s}$ decreases slowly for both increasing bar curvature radius and bar width. Therefore a smaller bar

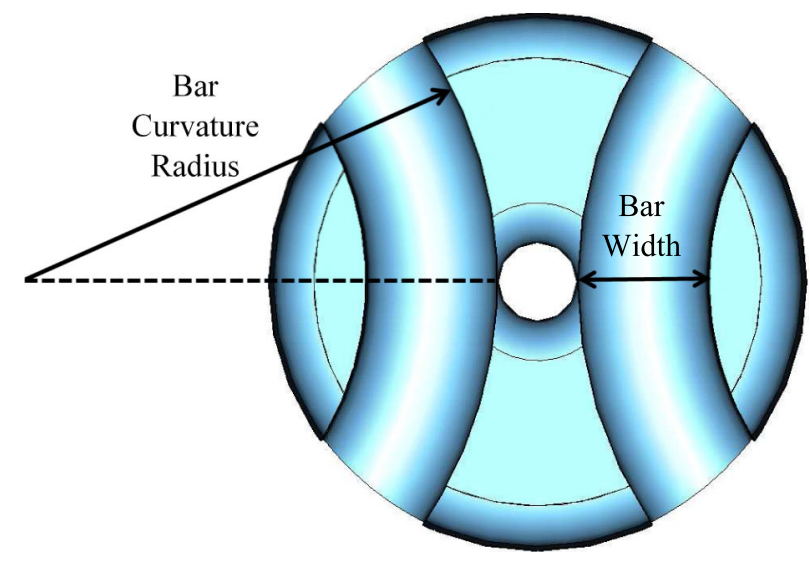

FIG. 18. Design parameters of optimization for the parallel-bar design with cylindrical outer conductor and curved race-trackshaped loading elements. 

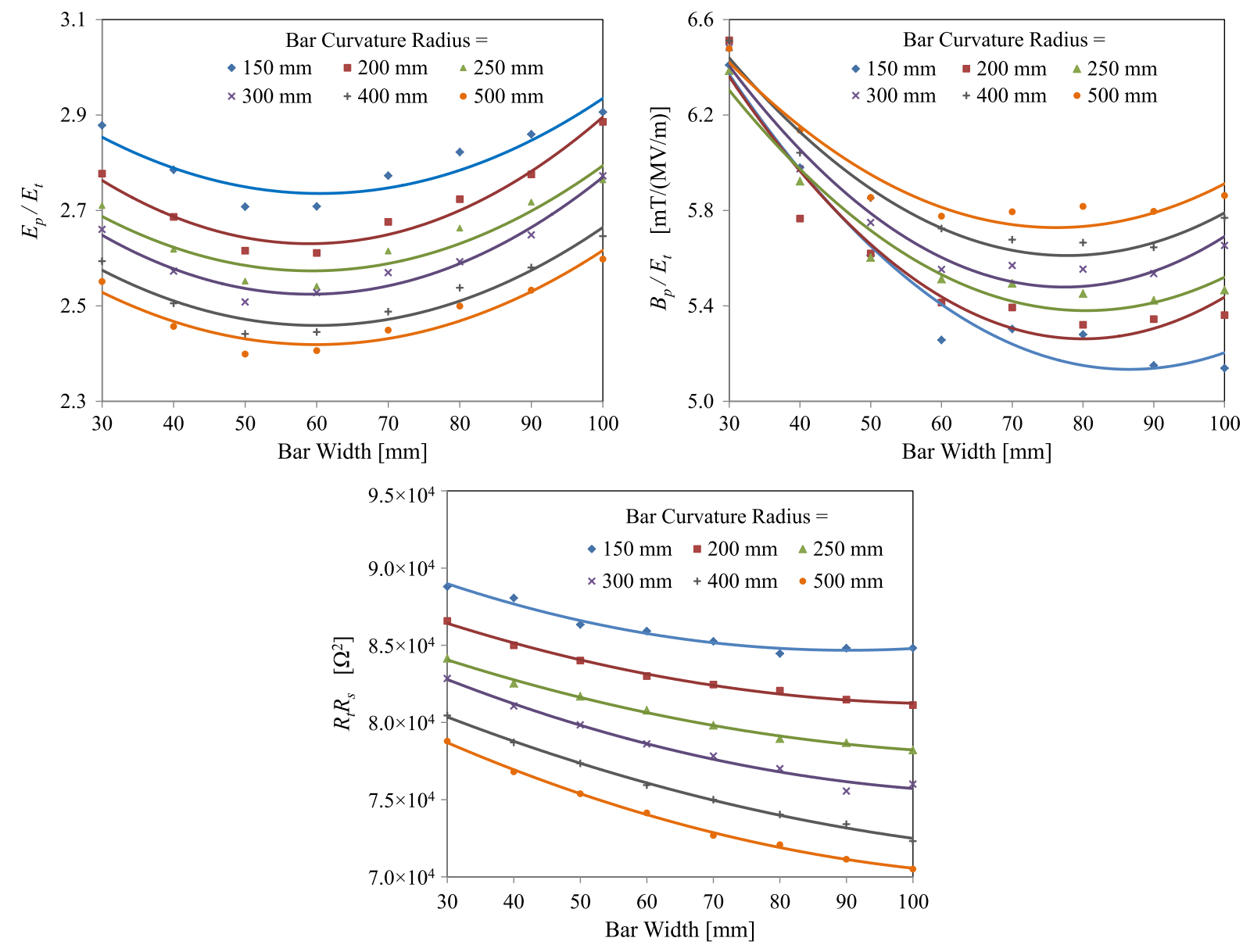

FIG. 19. Ratios of peak surface electric field $\left(E_{p}\right)$ and magnetic field $\left(B_{p}\right)$ to the transverse electric field $\left(E_{t}\right)$ and $R_{t} R_{s}$ with varying bar width and radius of bar curvature for race-track-shaped parallel bars [Design (D)].

curvature radius and a bar width is preferred to achieve higher shunt impedance.

In Designs (A), (B), and (C) the parallel-bar configuration is strictly parallel, where change in the bar separation will cause compensating changes in the inductance and capacitance, leaving the frequency almost constant. When the bars are curved as in Design (D) by keeping the bar separation constant, the inductance can be varied by changing the curvature of the bar surface. As the bars are curved, a change in separation causes different relative changes in capacitance and inductance, yielding a change in frequency.

The variation in peak surface electric and magnetic fields $\left(E_{p}\right.$ and $\left.B_{p}\right)$ for different bar widths and curvature radii, as shown in Fig. 20, gives the optimum bar width and the dependence on the peak field ratio $\left(B_{p} / E_{p}\right)$. The peak surface electric field can be reduced by increasing the bar curvature and the peak surface magnetic field can reduced by increasing the bar width. The two parameters of the bar width and curvature radii are optimized to reduce both



FIG. 20. Ratios of peak surface electric field $\left(E_{p}\right)$ and magnetic field $\left(B_{p}\right)$ to the transverse electric field $\left(E_{t}\right)$ with varying bar width and radius of bar curvature for race-track-shaped curved parallel bars [Design (D)]. 




FIG. 21. Design parameter of optimization for the parallel-bar design with bars merged onto cavity surface. surface electric and magnetic fields. As the bar width increases from $30 \mathrm{~mm}$, for all curvature radii, both peak electric and magnetic fields decrease and reach a minimum for a bar width of $\sim 60 \mathrm{~mm}$ beyond which they increase again. This clearly shows that eventually it is difficult to lower both peak fields simultaneously, only their ratio can be varied. The peak field ratio shown in Table I can be easily reduced to $\sim 2.0 \mathrm{mT} /(\mathrm{MV} / \mathrm{m})$. There is a significant increase in $R_{t} R_{s}$ due to the higher geometrical factor. Thus far, this design has the highest mode separation and the fundamental accelerating mode is no longer the lowest HOM mode.

In analyzing the HOM spectrum it was noticed that there are modes with field content only between the cavity outer surface and outer surface of the parallel bar while, in the deflecting mode, the fields are very small in that region. Therefore the parallel-bar design can be modified by increasing the bar width and merging it with the outer surface of the cavity as shown in Fig. 8: Design (E). This reduces
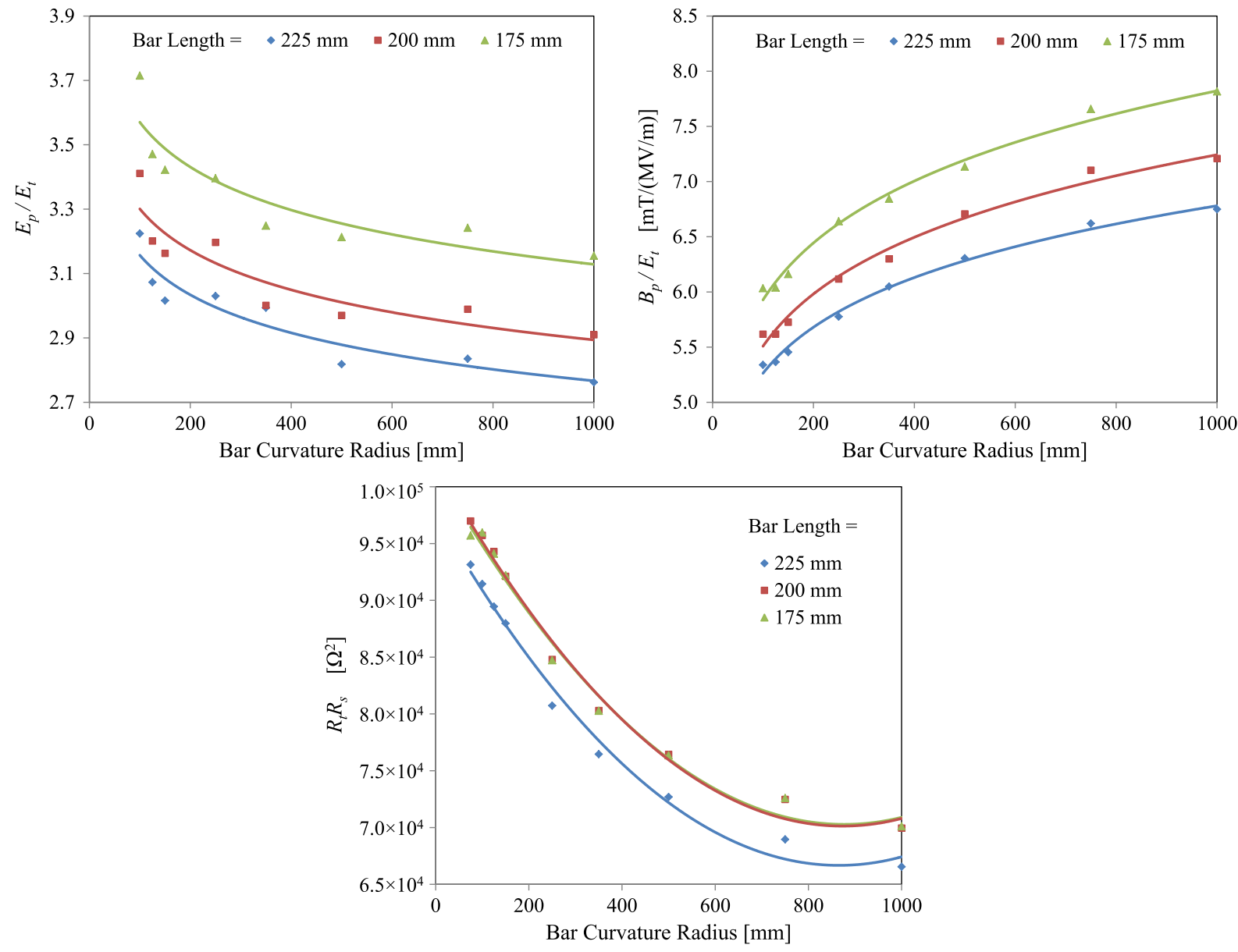

FIG. 22. Ratios of peak surface electric field $\left(E_{p}\right)$ and magnetic field $\left(B_{p}\right)$ to the transverse electric field $\left(E_{t}\right)$ and $R_{t} R_{s}$ with varying radius of bar curvature for parallel bars merged onto the cavity surface [Design (E)]. 


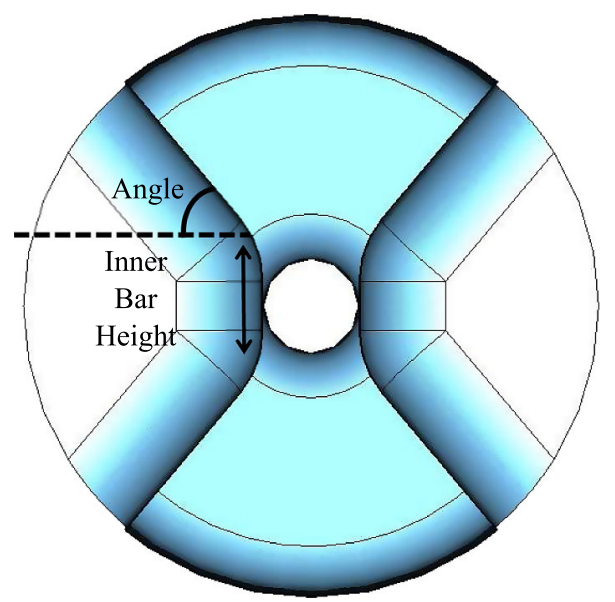

FIG. 23. Design parameters of optimization for the rf-dipole design with trapezoidal-shaped bars.
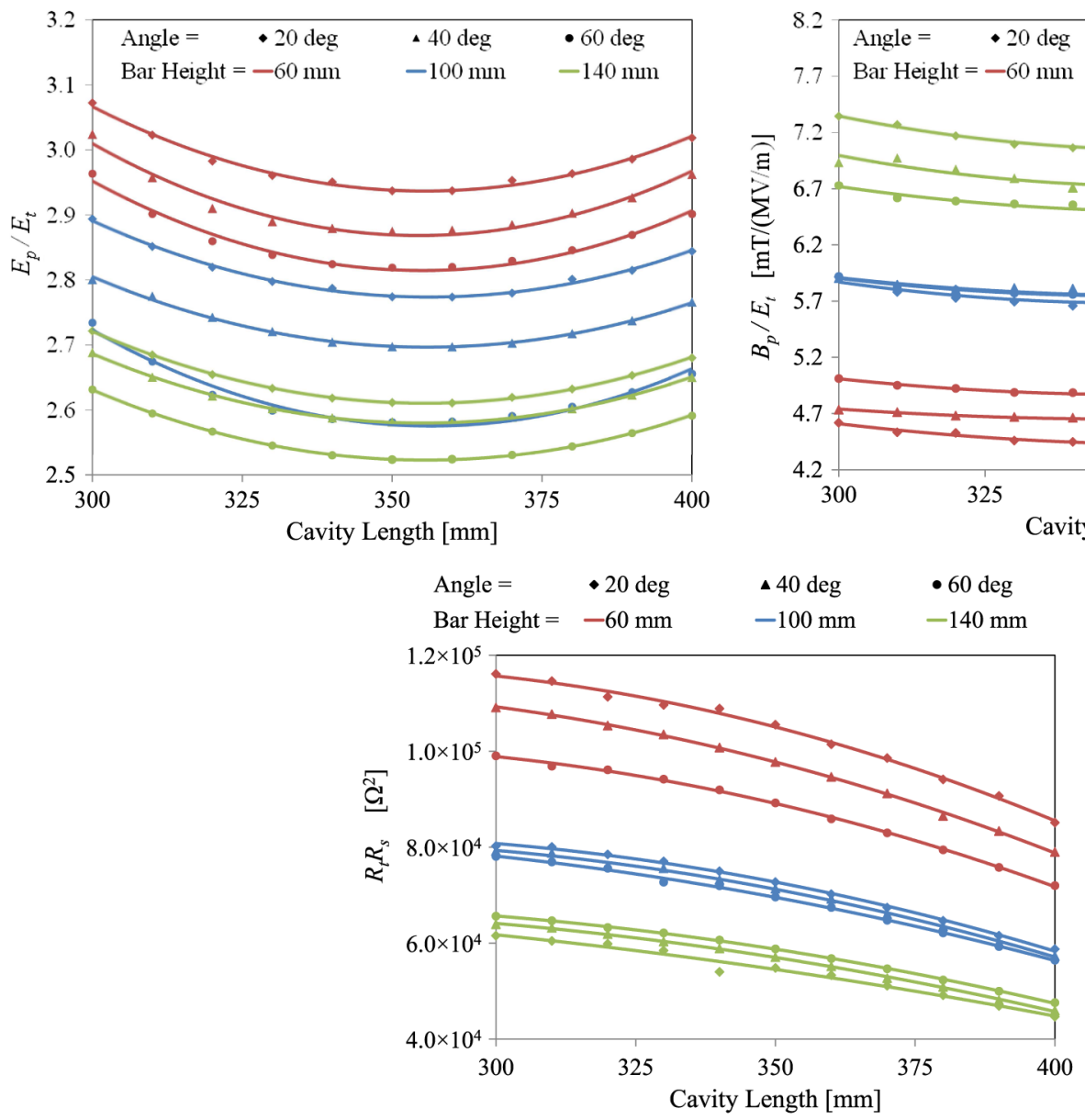

FIG. 24. Ratios of peak surface electric field $\left(E_{p}\right)$ and magnetic field $\left(B_{p}\right)$ to the transverse electric field $\left(E_{t}\right)$ and $R_{t} R_{s}$ with varying cavity length and bar length for different angles and inner bar heights of the trapezoidal-shaped parallel bars [Design (F)].

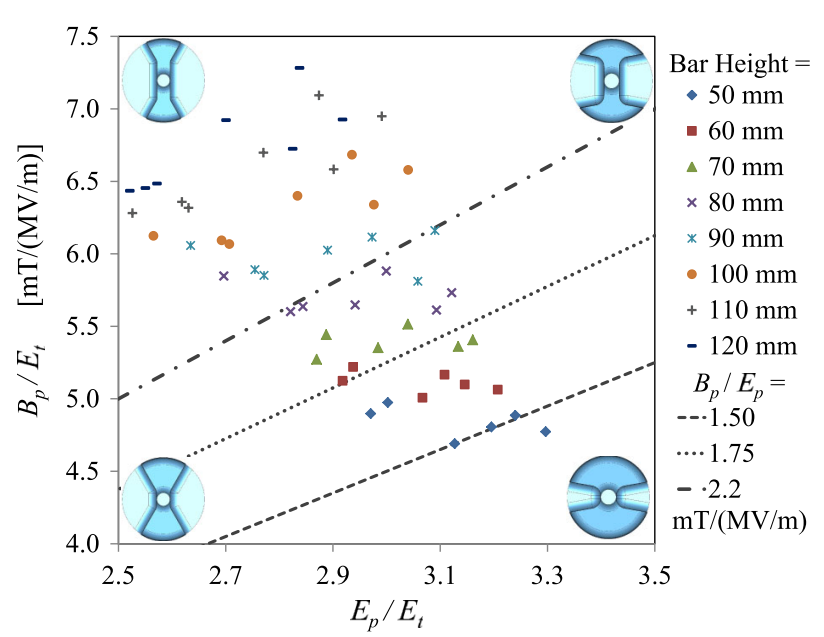

FIG. 25. Ratios of peak surface electric field $\left(E_{p}\right)$ and magnetic field $\left(B_{p}\right)$ to the transverse electric field $\left(E_{t}\right)$ with varying angle and inner bar height of the trapezoidal-shaped parallel bars [Design $(\mathrm{F})]$.





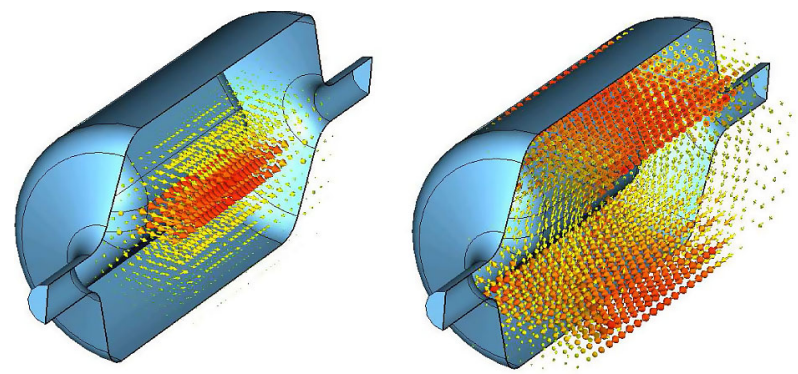

Electric Field

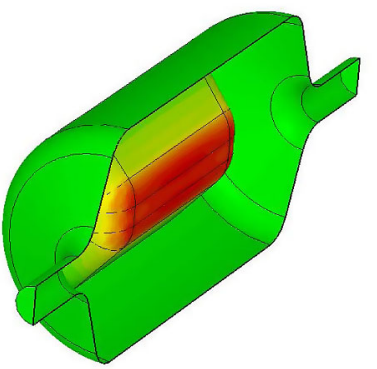

Surface Electric Field

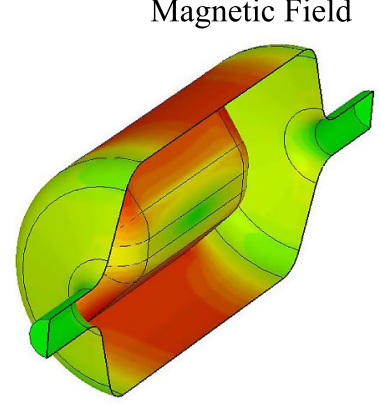

Surface Magnetic Field

FIG. 26. Field profile and peak surface fields of the rf-dipole cavity with cylindrical outer conductor and trapezoidal-shaped parallel bars [Design $(\mathrm{F})]$.

the number of HOMs in a given frequency range as the area between the outer conductor and the bars is reduced, the modes with field in that area disappear as their frequency goes to infinity. This also reduces the opportunity for multipacting in the deflecting mode [35]. With the merging of the walls, Design (E) becomes a design operating in a TE-like mode. In the transition from Design (D) to Design (E) the field components near the axis are absolutely identical and the modes of operation are the same.

The main parameter that optimizes the peak surface fields is the curvature of the bar (Fig. 21). The dependence of the curvature on the peak fields are shown in Fig. 22.

Keeping the bar separation constant and varying the curvature changes the inductance and therefore can be used to reduce the peak surface magnetic field. For smaller curvatures of bar radius the peak electric field is higher and drops faster as the curvature increases. In this case the peak surface magnetic field is relatively lower due to the wider spread of the surface fields. At larger radii of bar curvatures the peak surface magnetic field increases drastically as the bars are nearly vertical similarly to Design (D) of the parallel-bar cavity. The bar length and the cavity length are optimized to determine the optimum effective deflecting length. The properties of the optimized design, shown in Table I, are quite comparable with the properties of the previous design, but with a better HOM spectrum. The $R_{t} R_{s}$ is high at smaller bar curvature radii and drops faster as the curvature increase.

In the parallel-bar design with bars merged onto the cavity surface [Design (E): Fig. 8], the curvature controls the peak surface fields. This design restricts the optimization since it affects both surface fields simultaneously. Therefore the design is adapted further by varying the bar shape into a trapezoidal shape as shown in Design (F) in Fig. 8. The design has two key parameters: the inner bar height and angle as shown in Fig. 23 that are varied to control both peak surface fields independently. The cavity length and bar length are again changed simultaneously for different bar shapes to determine the optimum cavity length and bar length that minimize the surface fields as shown in Fig. 24. The peak surface electric field decreases with increasing inner bar height due to the increase in the surface area of the higher surface field. However, this increases the peak surface magnetic field as the magnetic field gets stronger at the top and the bottom of the cavity. The peak surface electric field is higher for smaller angles while the peak surface magnetic field is lower. The dependence of cavity length and the bar length on the peak surface fields are similar for different bar shapes.
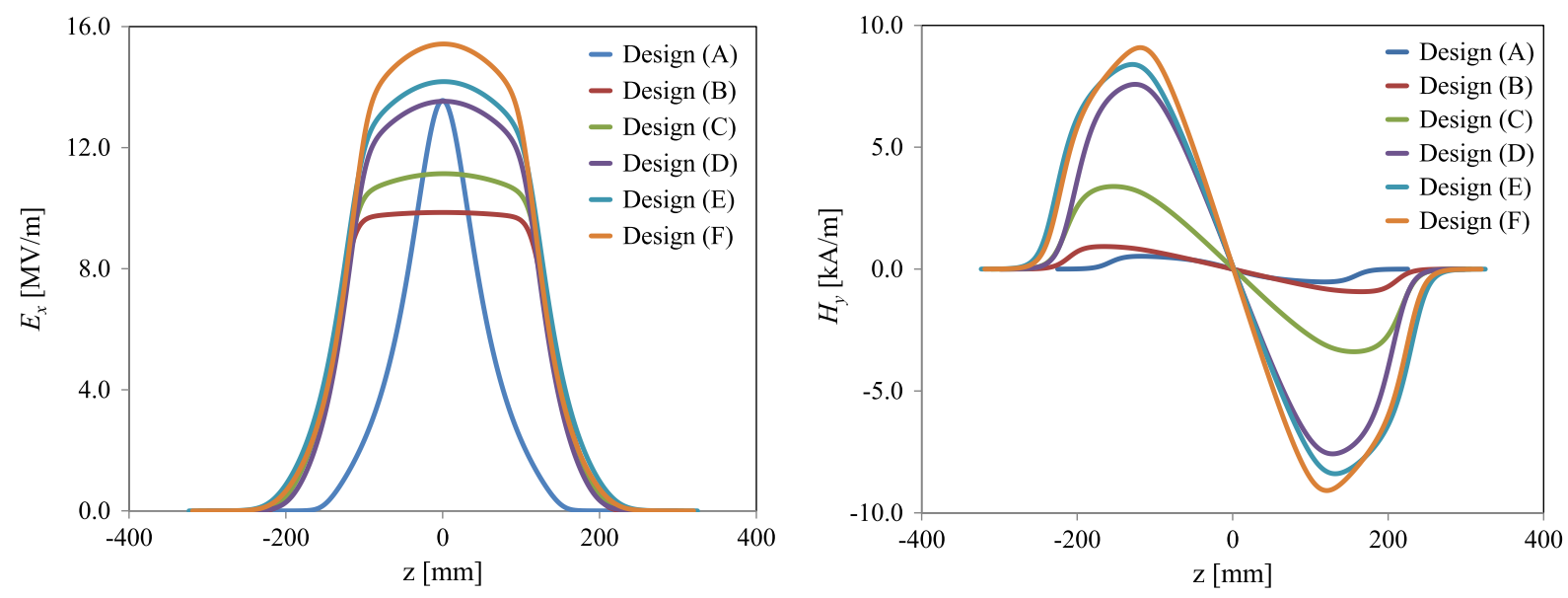

FIG. 27. On-axis electric (left) and magnetic (right) field of all parallel-bar designs shown in Fig. 8 normalized at a stored energy content of $1 \mathrm{~J}$. 

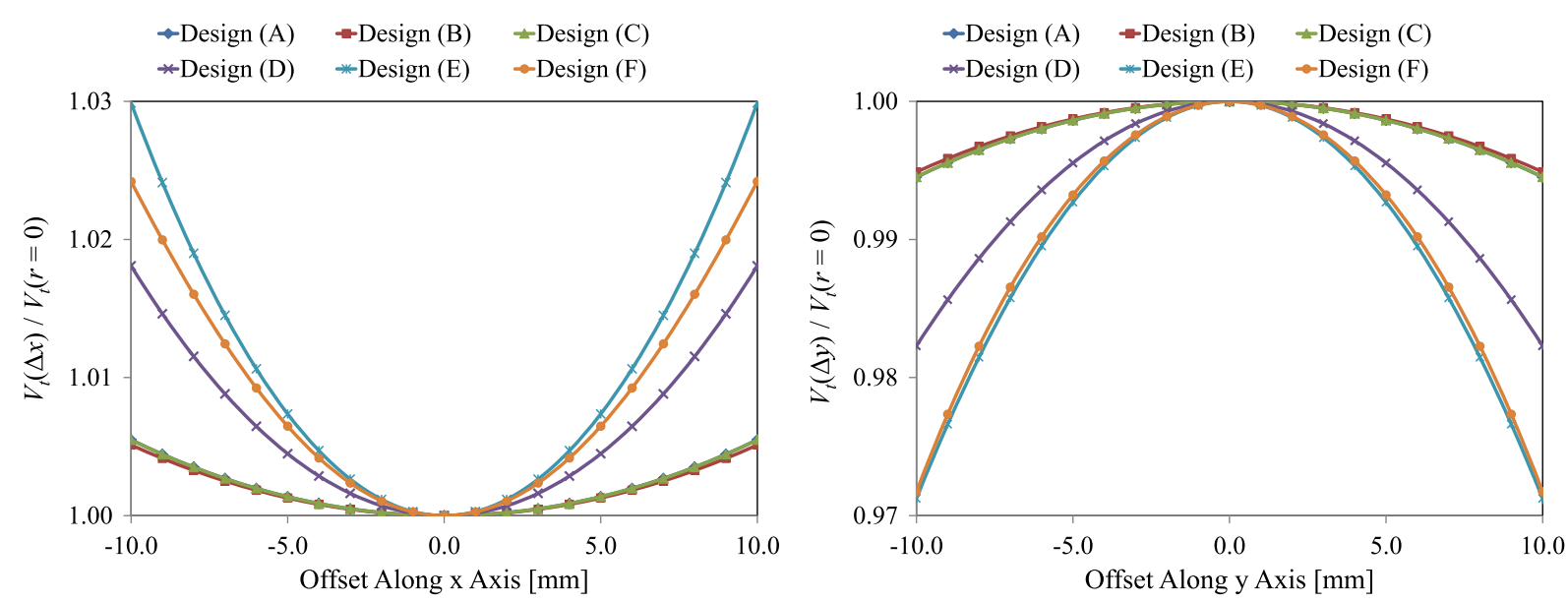

FIG. 28. Normalized transverse deflecting voltage in horizontal (offset along $x$ axis) and vertical (offset along $y$ axis) directions for designs shown in Fig. 8 [Designs (A) and (C) are identical].

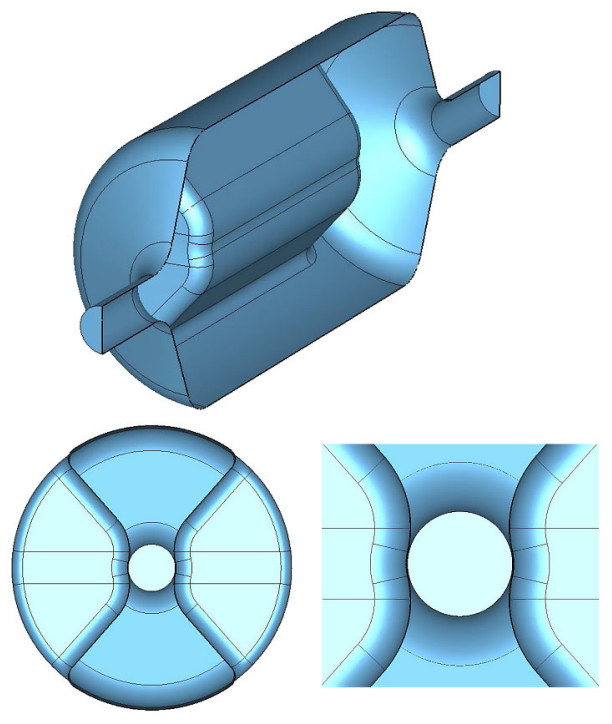

FIG. 29. Modified rf-dipole design with trapezoidal-shaped parallel bars indented in the beam line area.

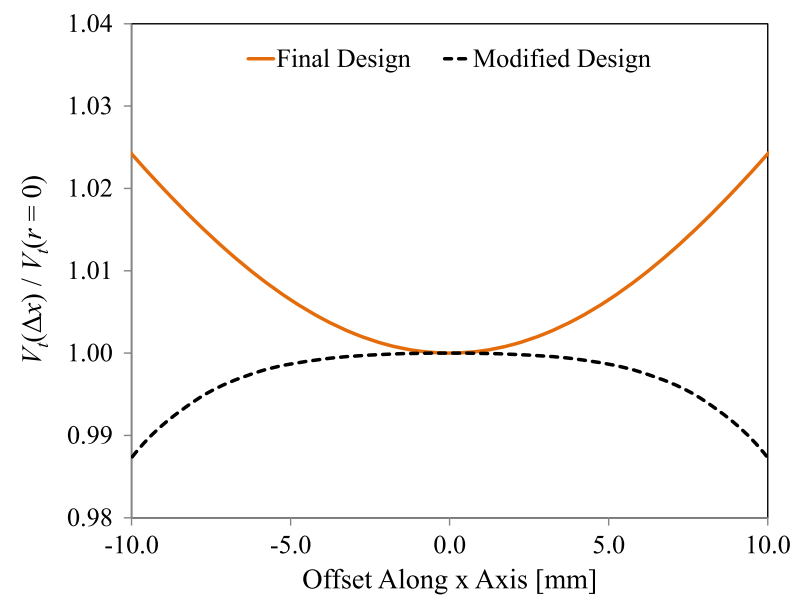

However, the change in peak surface electric field is higher than that of the peak surface magnetic field. The $R_{t} R_{s}$ is higher at smaller angles and at smaller bar heights. As the cavity length increases the $R_{t} R_{s}$ drops gradually.

The bar shape was optimized for different inner bar heights and angles to further minimize the peak surface fields with a balanced peak field ratio $\left(B_{p} / E_{p}\right)$. The $B_{p} / E_{p}$ decreases for smaller inner bar heights and larger angles as shown in Fig. 25. Reducing the bar height reduces the peak surface magnetic field, but increases the peak surface electric field and can be reduced by increasing the angle. However, the inner bar height is limited in this design by the chosen beam aperture diameter of $40 \mathrm{~mm}$ to maintain the field uniformity across the beam aperture. The trapezoidal-shaped bars have been curved appropriately to reduce field enhancement.

The properties of this design are shown in Table I. The peak surface electric field is invariant; however, the peak

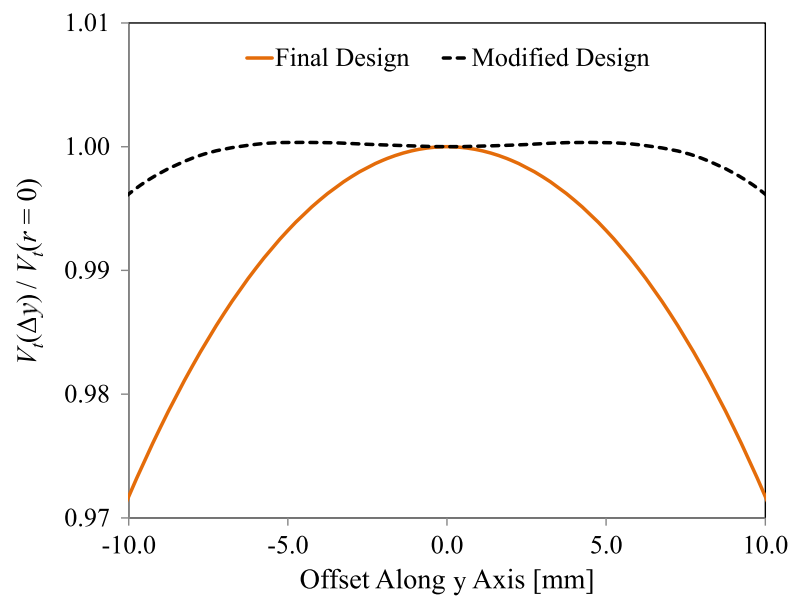

FIG. 30. Normalized transverse deflecting voltage in horizontal (offset along $x$ axis) and vertical (offset along $y$ axis) directions for Design (F) shown in Fig. 8 and modified rf-dipole design shown in Fig. 29. 


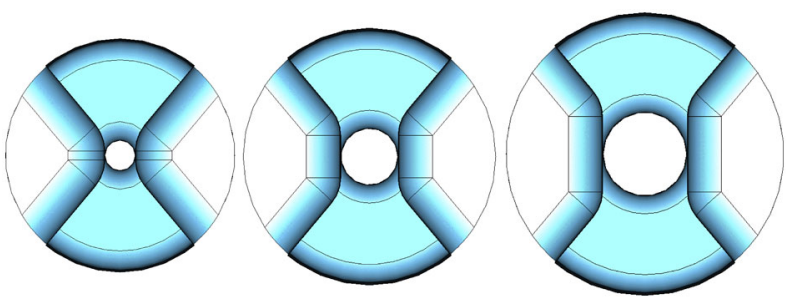

FIG. 31. RF-dipole cavity designs with trapezoidal-shaped parallel bars of varying beam aperture diameter and inner bar height for a given angle.

surface magnetic field is reduced by $16 \%$. For a final cavity design operating at $5 \mathrm{MV}$ of transverse voltage the peak surface electric field will be of $48 \mathrm{MV} / \mathrm{m}$ and $73 \mathrm{mT}$ of peak surface magnetic field with a peak field ratio of $1.53 \mathrm{mT} /(\mathrm{MV} / \mathrm{m})$.

The advantage of cylindrical-shaped design with trapezoidal bars is the ability to reduce surface magnetic fields with wider bars connecting to the outer wall. This design has the widest HOM spectrum compared to previous designs with a $278 \mathrm{MHz}$ separation between the $499 \mathrm{MHz}$ fundamental deflecting mode and the nearest higher-order mode. Furthermore the straight sections of inner bar height create a more uniform transverse electric field across the beam aperture. The design with the trapezoidal bars has the highest geometrical factor and $R_{t} R_{s}$, reducing the power dissipation in the walls. Additionally this design has the smallest transverse dimensions compared to other designs. The field profile and the surface field of optimized final geometry with cylindrical outer conductor and trapezoidal-shaped parallel bars are shown in Fig. 26. This geometry is essentially an rf-dipole geometry. It has some similarity with a room-temperature, multicell structure proposed by Paramonov et al. [36].

\section{B. Analysis of field nonuniformity}

In the designs shown in Fig. 8, the field profile on axis varies with each bar geometry as shown in Fig. 27. The field varies across the beam aperture off the beam axis generating nonuniform transverse deflection. In deflecting and crabbing cavities, in order to minimize emittance growth, the transverse deflection should be identical for all particles irrespective of their transverse position.

The transverse fields in both horizontal and vertical directions across the beam aperture are analyzed for all the designs as shown in Fig. 8. The designs with straight cylindrical or race-track-shaped loading elements have more uniform field across the beam aperture with variations of $0.5 \%$ as shown in Fig. 28 at an offset of $10 \mathrm{~mm}$. With more complicated loading elements the nonuniformity increases to less than $3 \%$ in both transverse directions.

The off-axis field variation follows a quadratic form within $\pm 10 \mathrm{~mm}$ radius as shown in Eq. (13) for Design (F) in Fig. 8:

$$
\frac{V_{t}(\Delta x, \Delta y)}{V_{t}(r=0)}=\left\{\begin{array}{l}
2.42 \times 10^{-4} \Delta x^{2}+1.0 \\
-2.83 \times 10^{-4} \Delta y^{2}+1.0 .
\end{array}\right.
$$

In the rf-dipole design with trapezoidal-shaped loading elements the height of the inner wall is reduced to lower the peak electric field. However, this increases the nonuniformity across the beam aperture. The nonuniformity can be reduced by curving the beam aperture inwardly as shown in Fig. 29. The nonuniformity in the transverse field is almost completely canceled across the beam aperture within the transverse beam size for a design with an inner bar surface moved inward around the beam aperture by a distance of $5 \mathrm{~mm}$. A field nonuniformity analysis was carried out for this design, evaluating the field across the beam aperture as shown in Fig. 30 to determine the change in transverse voltage for both horizontal and vertical directions. The nonuniformity can be reduced by increasing the

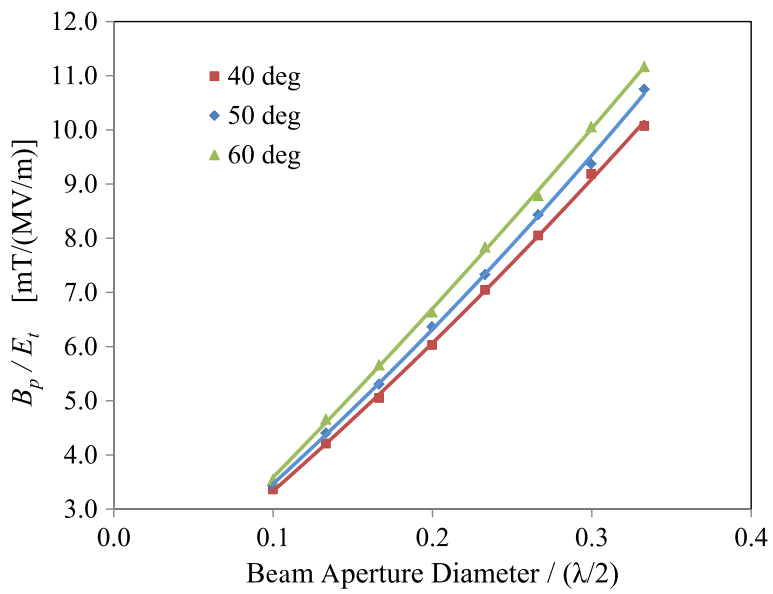

FIG. 32. Ratios of peak surface electric field $\left(E_{p}\right)$ and magnetic field $\left(B_{p}\right)$ to the transverse electric field $\left(E_{t}\right)$ with varying beam aperture diameter and angle for rf-dipole design with trapezoidal-shaped bars. 


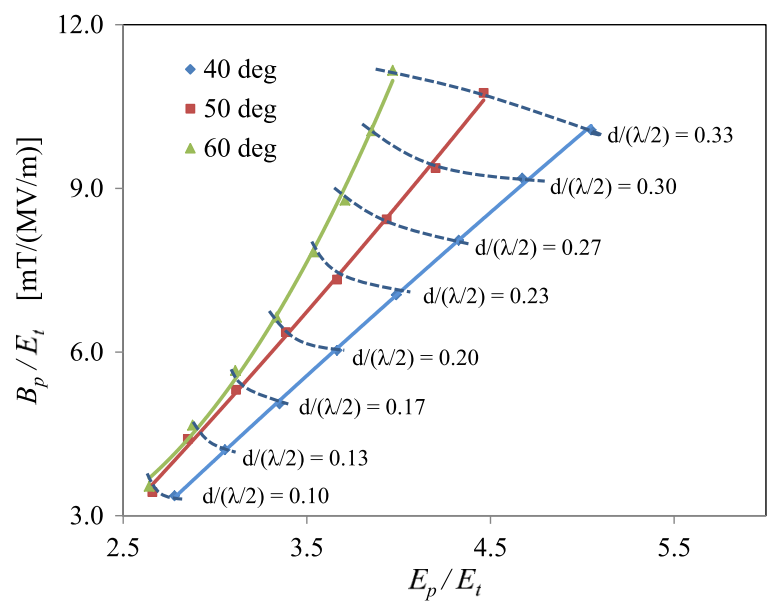

FIG. 33. Ratios of peak surface magnetic field $\left(B_{p}\right)$ to the electric field $\left(E_{p}\right)$ with varying beam aperture diameter and angle for rf-dipole design with trapezoidal-shaped bars.

inner bar height and/or by giving it a curved shape in the beam line region. We have investigated so far only a circular indentation of the bars in the beam line region. Further optimization could lead to even better uniformity of the deflecting voltage off axis.

\section{Dependence of beam aperture on design properties}

The parallel-bar rf-dipole designs can be used for many deflecting/crabbing cavity applications due their compactness and attractive properties in low peak surface fields and higher net deflection and the absence of lower-order modes while the frequency of the nearest higher-order mode is of the order of 1.5 times that of the fundamental. The diameter of the beam aperture and the design frequency are the two important parameters in designing parallel-bar rfdipole geometries. The design can be scaled to obtain the required design frequency as the frequency is inversely

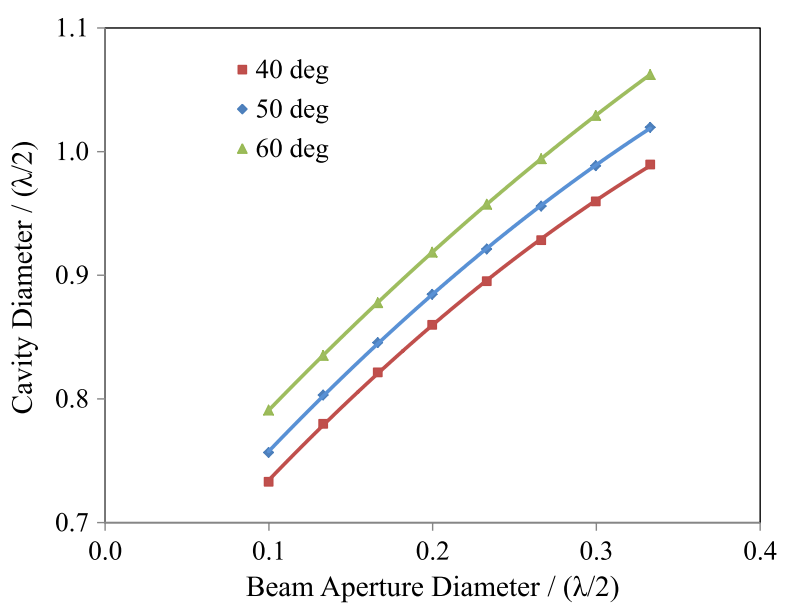

FIG. 34. Cavity diameter with varying beam aperture diameter and angle for rf-dipole design with trapezoidal-shaped bars. related to the cavity dimensions. The $499 \mathrm{MHz}$ rf-dipole cavity with trapezoidal-shaped loading elements and cylindrical outer conductor was also analyzed for varying beam aperture radius and angle of the trapezoidal-shaped parallel bars by adjusting the inner bar height proportionally (Fig. 31).

The dependence of $E_{p} / E_{t}$ and $B_{p} / E_{t}$ on the beam aperture is shown in Fig. 32. As expected the peak fields increase as the beam aperture is increased. The $B_{p} / E_{p}$ shown in Fig. 33 gives the peak field ratio for different beam aperture diameter normalized to the half wavelength of the cavity, and different angles of the trapezoidal-shaped bars. The dotted lines show the constant ratios of normalized beam aperture diameter. Therefore the angle can be used to set the peak field ratio as needed by the design parameters for a given design frequency and beam aperture diameter. As shown in Fig. 34 larger beam aperture radii make the design geometries to have large cavity radii.

The transverse electric field ( $\left.E_{t, \text { Total }}\right)$ determined using both on-axis electric and magnetic field components as given in Eqs. (5) and (7) is compared to the contribution only from the on-axis transverse electric field $\left(E_{t, E \text { Only }}\right)$ with varying beam aperture diameter. The $E_{t, \text { Total }} / E_{t, E \text { Only }}$ dependence on the normalized beam aperture diameter to the half wavelength of the cavity is shown in Fig. 35. As the beam aperture is increased the deflecting voltage due to electric field on axis decreases faster than the deflecting voltage due to the on-axis magnetic field. Therefore the negative contribution from the magnetic field is larger at large aperture reducing the net deflecting voltage. The ratio approaches zero as the beam aperture diameter approaches the cavity diameter; this would correspond to a cylindrical cavity operating in the $\mathrm{TE}_{111}$ mode which, according to the Panofsky-Wenzel theorem $[18,19]$, produces no deflecting voltage. The magnetic field contribution is directly due to the on-axis magnetic field component with the fields

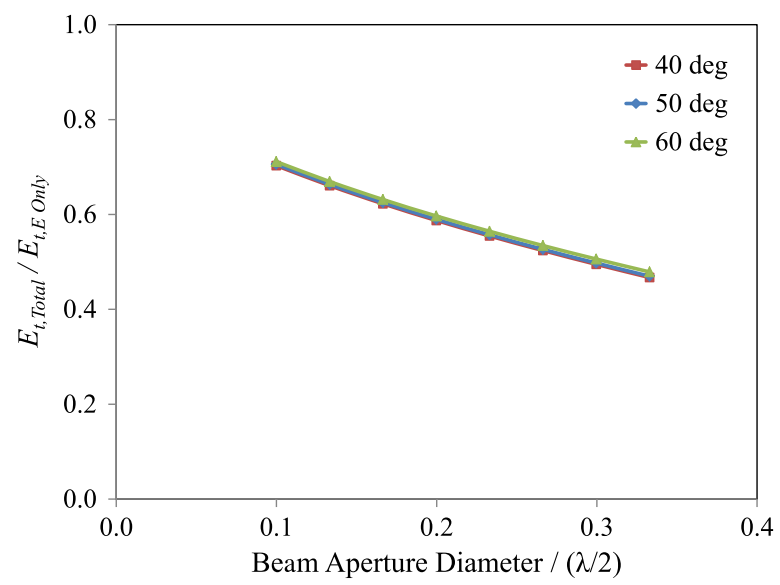

FIG. 35. Electric field and magnetic field contribution to the transverse deflection of the rf-dipole cavity designs with the varying beam aperture. 

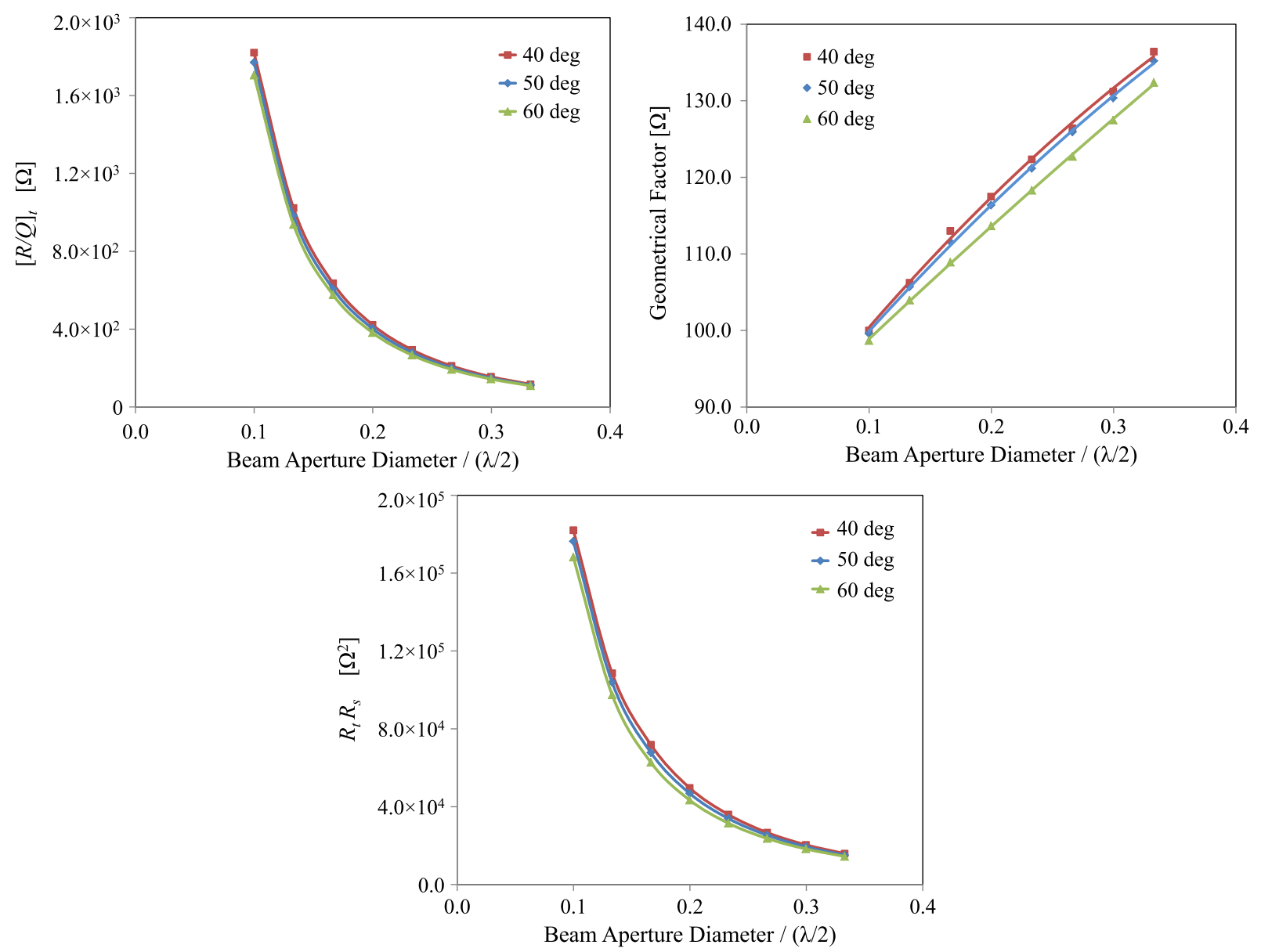

FIG. 36. $R_{t} / Q$, geometrical factor $G=Q R_{s}$, and $R_{t} R_{s}$ as function of the beam line aperture for rf-dipole design with trapezoidalshaped bars.

confined between the end plates and bars. Since the slope of the end plates and the bar length have not been changed while scaling the designs for different aperture, the contribution on $E_{t, \text { Total }} / E_{t, E \text { Only }}$ with beam aperture variation has little dependency on the angle of the trapezoidalshaped bars.

The $[R / Q]_{t}$ drops drastically as the beam aperture increases. Irrespective of the increase in geometrical factor $(G)$, the resulting $R_{t} R_{s}$ also decreases in the order of $d^{2}$ with the beam aperture diameter $(d)$ as shown in Fig. 36.

\section{CONCLUSION}

The parallel-bar rf-dipole deflecting/crabbing structure has been optimized and a number of variations have been analyzed and presented. The rf-dipole design with trapezoidal-shaped bars geometry with the cylindrical outer conductor has been shown to have significantly improved properties compared to other geometries. This geometry is capable of delivering lower and well-balanced peak surface fields with high shunt impedance. Another attractive feature is the fact that this geometry has no lower-order mode and the nearest higher-order mode is far removed from the fundamental mode. The shape of the bars connecting to the outer conductor with sloped end plates adds rigidity to the design in terms of mechanical deformations. We have presented here the optimization studies for a $499 \mathrm{MHz}$ deflecting cavity for the Jefferson Lab $12 \mathrm{GeV}$ upgrade; similar studies have also been done for a $400 \mathrm{MHz}$ crabbing cavity for the LHC luminosity upgrade. Both of these cavities are now being fabricated. The results of the analysis of the HOM properties, multipole expansion of the rf fields, fabrication of prototypes, and the experimental results of the parallel-bar rf-dipole geometries will be the subject of later publications. Furthermore, detailed analysis on cavity sensitivity to microphonics, Lorentz force detuning, and cavity tuning will be presented in another publication with simulation results and measurement data obtained for the prototypes of the rf-dipole cavity.

\section{ACKNOWLEDGMENTS}

We want to acknowledge the fruitful discussions with Zenghai Li of SLAC who had arrived at a similar rf-dipole geometry from a completely different starting point, 
namely the ridged waveguide design. This manuscript has been partially authored by Jefferson Science Associates, LLC under U.S. DOE Contract No. DE-AC0506OR23177. This work was also partially supported by the Small Business Technology Transfer (STTR) Program and by the U.S. LHC Accelerator Research Program (LARP) through U.S. Department of Energy Contracts No. DE-AC02-07CH11359, No. DE-AC02-98CH10886, No. DE-AC02-05CH11231, and No. DE-AC0276 SF00515.

[1] P. R. Phillips, Rev. Sci. Instrum. 32, 13 (1961).

[2] M. Bell, P. Bramham, and B.W. Montague, Nature (London) 198, 277 (1963).

[3] O. H. Altenmueller, R. R. Larsen, and G. A. Loew, Rev. Sci. Instrum. 35, 438 (1964).

[4] H. Hahn and H. J. Halama, Rev. Sci. Instrum. 36, 1788 (1965).

[5] H. Hahn, Rev. Sci. Instrum. 34, 1094 (1963).

[6] A. Citron, G. Dammertz, M. Grundner, L. Husson, R. Lehm, H. Lengeler, D. E. Plane, and G. Winkler, Nucl. Instrum. Methods 155, 93 (1978).

[7] C. W. Leemann and C. G. Yao, in Proceedings of the 1990 Linear Accelerator Conference, Albuquerque, New Mexico (Los Alamos Report No. LA-12004-C, 1991), p. 232.

[8] Y. Senichev, O. Belyaev, W. Bräutigam, Y. Budanov, R. Maier, V. Stepanov, V. Teplyakov, A. Zherebtsov, and I. Zvonarev, Phys. Rev. ST Accel. Beams 9, 012001 (2006).

[9] R. B. Palmer, SLAC Technical Report No. SLAC-PUB4707, 1988.

[10] K. Oide and K. Yokoya, Phys. Rev. A 40, 315 (1989).

[11] K. Hosoyama, K. Hara, A. Kabe, Y. Kojima, Y. Morita, H. Nakai, L. S. Peng, K. Ohkubo, H. Hatori, and M. Inoue, in Proceedings of the 1998 Asian Particle Accelerator Conference, Tsukuba, Japan (KEK Proceedings 98-10, 1998), p. 828.

[12] R. Calaga, in Proceedings of the 2011 Conference on RF Superconductivity (Chicago, Illinois, 2011), p. 988.

[13] Z. Li, L. Xiao, C. Ng, and T. Markiewicz, in Proceedings of the 2010 International Particle Accelerator Conference (IPAC'10 OC/ACFA, Kyoto, Japan, 2010), p. 504.

[14] J. R. Delayen and H. Wang, Phys. Rev. ST Accel. Beams 12, 062002 (2009).

[15] B. Hall, G. Burt, C. Lingwood, R. Rimmer, and H. Wang, in Proceedings of the 2010 International Particle Accelerator Conference (Ref. [13]), p. 3001.

[16] J. R. Delayen, in Proceedings of the 2011 Conference on RF Superconductivity (Ref. [12]), p. 631.

[17] I. Ben-Zvi, in Proceedings of the 2011 Conference on RF Superconductivity (Ref. [12]), p. 637.

[18] W. K. H. Panofsky and W. A. Wenzel, Rev. Sci. Instrum. 27, 967 (1956).

[19] M.J. Browman, in Proceedings of the 1993 Particle Accelerator Conference, Washington, DC (IEEE, New York, 1993), p. 800.
[20] H. Padamsee, P. Barnes, C. Chen, J. Kirchgessner, D. Moffat, D. Rubin, Y. Samed, J. Sears, Q. Shu, M. Tigner, and D. $\mathrm{Zu}$, in Proceedings of the 1991 Particle Accelerator Conference, San Francisco, California (IEEE, New York, 1991), p. 786.

[21] T.W. Koeth, in Proceedings of the 1999 Particle Accelerator Conference, New York, New York (IEEE, New York, 1999), p. 995.

[22] J. Shi, H. Chen, S. Zheng, J. M. Byrd, and D. Li, in Proceedings of the 2005 Particle Accelerator Conference, Knoxville, Tennessee (IEEE, New York, 2005), p. 4287.

[23] G. Waldschmidt, B. Brajuskovic, A. Nassiri, G. Cheng, J. Henry, J. Mammosser, R. Rimmer, and H. Wang, in Proceedings of the 2011 Particle Accelerator Conference, New York (IEEE, New York, 2011), p. 2516.

[24] C. Hovater, G. Arnold, J. Fugitt, L. Harwood, R. Kazimi, G. Lahti, J. Mammosser, R. Nelson, C. Piler, and L. Turlington, in Proceedings of the 1996 Linear Accelerator Conference, Geneva, Switzerland (CERN Report No. 96-07, 1996), p. 77.

[25] H. Padamsee, J. Knobloch, and T. Hays, RF Superconductivity for Accelerators (Wiley-VCH, Germany, 2008).

[26] H. Padamsee, RF Superconductivity: Volume II: Science, Technology and Applications (Wiley-VCH, Germany, 2009).

[27] P. N. Ostroumov, B. Mustapha, Z. A. Conway, R. L. Fischer, S. Gerbick, M. Kedzie, M. P. Kelly, I. V. Gonin, and S. Nagaitsev, in Proceedings of the 2012 International Particle Accelerator Conference, New Orleans, Louisiana, USA (IPAC'12 OC/IEEE, 2012), p. 2295.

[28] J. R. Delayen and S. U. De Silva, in Proceedings of the 2011 Particle Accelerator Conference, New York (Ref. [23]), p. 1021.

[29] J.R. Delayen and S. U. De Silva, in Proceedings of the 2011 Conference on RF Superconductivity (Ref. [12]), p. 219.

[30] Microwave Studio, CST GmbH, Darmstadt, Germany [http://www.cst.com].

[31] S. U. De Silva and J.R. Delayen, in Proceedings of the 2010 International Particle Accelerator Conference (Ref. [13]), p. 3075.

[32] S. U. De Silva and J.R. Delayen, in Proceedings of the 2011 Conference on RF Superconductivity (Ref. [12]), p. 135.

[33] S. U. De Silva and J.R. Delayen, in Proceedings of the 2009 Conference on RF Superconductivity (HZBBerichte, Berlin, Germany, 2009), p. 589.

[34] S. U. De Silva and J.R. Delayen, in Proceedings of the 2010 Linear Accelerator Conference (LINAC10 OC, Tsukuba, Japan, 2010), p. 812.

[35] S. U. De Silva and J.R. Delayen, in Proceedings of the 2011 Particle Accelerator Conference, New York (Ref. [23]), p. 1018.

[36] V. Paramonov, L. Kravchuk, and S. Korepanov, in Proceedings of the 2006 Linear Accelerator Conference (LINAC06 OC, Knoxville, Tennessee, 2006), p. 649. 